\title{
Review Article \\ Screening for Precancerous Lesions of Upper Gastrointestinal Tract: From the Endoscopists' Viewpoint
}

\author{
Chen-Shuan Chung ${ }^{1,2}$ and Hsiu-Po Wang ${ }^{1}$ \\ ${ }^{1}$ Department of Internal Medicine, National Taiwan University Hospital, College of Medicine, National Taiwan University, \\ Chung-Shan South Road, Taipei 100, Taiwan \\ ${ }^{2}$ Department of Internal Medicine, Far Eastern Memorial Hospital, Banciao District, New Taipei City, Taiwan
}

Correspondence should be addressed to Hsiu-Po Wang; wanghp@ntu.edu.tw

Received 3 November 2012; Accepted 19 February 2013

Academic Editor: Fayez Sandouk

Copyright $\odot 2013$ C.-S. Chung and H.-P. Wang. This is an open access article distributed under the Creative Commons Attribution License, which permits unrestricted use, distribution, and reproduction in any medium, provided the original work is properly cited.

Upper gastrointestinal tract cancers are one of the most important leading causes of cancer death worldwide. Diagnosis at late stages always brings about poor outcome of these malignancies. The early detection of precancerous or early cancerous lesions of gastrointestinal tract is therefore of utmost importance to improve the overall outcome and maintain a good quality of life of patients. The desire of endoscopists to visualize the invisibles under conventional white-light endoscopy has accelerated the advancements in endoscopy technologies. Nowadays, image-enhanced endoscopy which utilizes optical-or dye-based contrasting techniques has been widely applied in endoscopic screening program of gastrointestinal tract malignancies. These contrasting endoscopic technologies not only improve the visualization of early foci missed by conventional endoscopy, but also gain the insight of histopathology and tumor invasiveness, that is so-called optical biopsy. Here, we will review the application of advanced endoscopy technique in screening program of upper gastrointestinal tract cancers.

\section{Introduction}

Malignancies of gastrointestinal tract represent the leading cause of cancer death worldwide. Esophageal and gastric cancers, which have an overall 5-year survival rate of $10 \sim 20 \%$ and $20 \sim 30 \%$, respectively, comprise the majority of the upper gastrointestinal (UGI) tract malignancy $[1,2]$. Barrett's esophagus (BE) was regarded as the premalignant lesion for esophageal adenocarcinoma (EAC) based on the unmasking of underlying intestinal metaplasia mucosa after chemotherapy [3]. Moreover, a retrospective study has shown high prevalence of $\mathrm{BE}$ in pretreatment $(75 \%)$ and postchemotherapy (97\%) EAC patients [4]. However, emerging evidence has shown that the risk of EAC in BE patients was not as high as expected previously [5]. Recently, a nationwide populationbased cohort study disclosed that the annual risk of EAC was $0.12 \%$ among patients with $\mathrm{BE}$ (increased to $0.26 \%$ when high-grade dysplasia was taken into account) [5]. The results call into question the rationale for periodically endoscopic surveillance in BE patients without dysplasia. Unlike the higher proportion of the histology subtype adenocarcinoma of the esophagus in Western countries, approximately over $90 \%$ of the esophageal cancer in countries located at the "esophageal cancer belt," which stretches from Caspian Sea across Central Asia to the West Pacific, are esophageal squamous cell carcinomas (ESCC) [1]. The global incidence of esophageal cancer increased gradually probably because of increasing prevalence of the $\mathrm{BE}$ and the habits of psychoactive substance use, such as cigarettes smoking, alcohol consumption, and betel quid chewing, which cause ESCC [1]. Conversely, a steady declining incidence of gastric cancer in many countries has been observed in the last few decades probably because of improved sanitation and Helicobacter pylori (Hp) eradication therapy $[1,2]$. Hp infection, atrophic gastritis, and intestinal metaplasia are among the most important premalignant conditions of gastric cancer $[1,6]$. In patients at risk, annual surveillance can detect tumors at an earlier stage (stage I and II 67\% versus $23 \%$; $P<0.05$ ) with 
a major improvement in 5-year survival (50\% versus 10\%; $P=$ 0.006 ) when compared with open access study population [6]. The UGI tract cancer remains imposing considerable impacts on the public health.

The outcome of UGI tract cancer is closely associated with the stage at diagnosis. The poor prognosis of UGI tract cancer is largely attributed to the delay diagnosis at symptomatic conditions. Nevertheless, the 5-year survival rate for superficial ESCC and early gastric cancer (EGC) may exceed $80 \%$ and $90 \%$, respectively [1, 7-9]. Based on an investigation of 290 surgically resected ESCC cases, cancers invading epithelium and lamina propria did not have lymph node (LN) metastasis, and a 5-year survival rate for surgical resected cancers limited to mucosa, and superficial submucosa was $100 \%$ [9]. The accumulated experience has also shown that EGC has lower risk for LN metastasis. The analysis of over thousands of gastric cancer patients who had undergone gastrectomy and LN dissection has suggested the low risk of LN metastasis for well-differentiated intramucosal adenocarcinoma without ulcer findings irrespective of the tumor size and those less than $3 \mathrm{~cm}$ with ulcer findings $[10,11]$. Neither of well-differentiated EGC invading superficial $(<500 \mu \mathrm{m})$ submucosa with tumor size less than $3 \mathrm{~cm}$ and undifferentiated intramucosal EGC less than $2 \mathrm{~cm}$ without ulcers have LN metastasis risk [10, 11]. The clinical experiences and data disclosed excellent survivals for these early UGI tract cancers as long as the potential for LN metastasis can be excluded $[1,2,7,9]$.

In the last few decades, endoscopists try to use minimally invasive procedures to remove the superficial UGI tract cancers without concern of LN metastasis. The survival of early cancers after endoscopic removal with organ preservation could be similar to those after surgical resection. Endoscopic removal of EGC was first described in 1974, and endoscopic mucosal resection (EMR) technique with "strip-biopsy" method was published in 1984 [12, 13]. In the following years, different EMR techniques with improvement in equipment accessories, such as cap and ligation method, have been introduced $[14,15]$. However, the en bloc resection rate using EMR methods was low, especially for those larger than $2 \mathrm{~cm}$ or with invasion deeper than submucosa layer which were prone to recur locally even after piecemeal EMR $[13,16]$. To achieve minimal invasive en bloc resection and provide intact specimen for pathological examination, endoscopic submucosal dissection (ESD) becomes a safe alternative for endoscopic management of early UGI tract cancers $[1,13,16,17]$. The en bloc rate and recurrence rate of ESD for early ESCC were about 95\% and 0\%, respectively [16]. Similarly, the en bloc rate for EGC in the ESD group was significantly higher than that in EMR group (odds ratio (OR) 9.69; 95\% confidence interval (CI), 7.74-12.13), and the ESD group had lower recurrence rate (OR 0.10; 95\% CI, 0.06-0.18) [18]. Therefore, since the early 2000s, ESD has been regarded as a treatment alternative for early UGI tract cancers in most Asian countries, including Japan, Korean, China, and Taiwan.

Given the high chance for curative treatment of early UGI tract cancers by endoscopic resection with intents of minimal invasiveness, a detailed and thorough endoscopic examination for precancerous or early cancerous lesions is of paramount importance to improve the overall outcome. Here, we will review the literatures on the surveillance of precancerous lesions of UGI tract from the endoscopist viewpoint.

\section{Image-Enhanced Endoscopy Screening of Upper Gastrointestinal Tract Cancers}

The subtle change from precancerous or early cancerous UGI tract cancers is always challenging to endoscopists when using conventional white-light imaging (WLI) endoscopy. To enhance the contrast and resolution of endoscopic images, advances in biomedical optics and endoscopic equipments have been made rapidly in the latest decade. The imageenhanced endoscopy (IEE) system utilizes different enhancing methods by means of dye, optical, and electronic contrasting to allow improved visualization of precancerous lesions and to gain insight of the pathology and invasiveness of the lesions [19]. The techniques and applications of IEE in surveillance of UGI tract cancer will be described.

\subsection{The Principles of IEE for UGI Tract}

2.1.1. Narrow-Band Imaging System with Magnifying Endoscopy. The development of spectroscopy began over 10 years ago. Many efforts have been made to find the best specific pattern of spectrum for excellent enhancement of mucosa surface and microvascular architecture. The narrow-band imaging (NBI) system which was developed since 1999 and commercialized available in 2005. In NBI system, an optic filter is used to illuminate lights with $400-430 \mathrm{~nm}$ and 525-555 nm narrowed wavelengths instead of red-green-blue (RGB) broadband light which is utilized in conventional WLI system. Based on the characteristics of light absorption and scattering, the NBI system can accentuate the mucosa surface and vessels at different depths [20]. Angiogenesis is one of the subtle histological changes during carcinogenesis. The hemoglobin which can be considered as a chromophore associated with angiogenesis plays an important role in image formation of living tissues. The GB light is well absorbed by the hemoglobin, thereby contrasting well the morphology of vessels at different depths. The superficial vessels absorb light, with shorter wavelength and become reddish brown, which is the complementary color of GB light, whereas the deeper vessels absorb light with longer wavelength and appear cyanic hue (Figure 1). It is most appropriate to use $415 \pm 15 \mathrm{~nm}$ blue light and $540 \pm 15 \mathrm{~nm}$ green light to observe mucosal surface microvasculature and deeper submucosal vessel, respectively. Magnifying or zoom endoscope has been developed over 40 years [21]. Combined with NBI system, the magnifying endoscope (ME) which has the ability to magnify the image to $150 \mathrm{x}$ with minimal discriminating diameter about $10 \mu \mathrm{m}$ can detect precancerous or early cancerous lesions more accurately $[22,23]$. For the well-fixed magnified images, a black rubber or transparent plastic hood is crucial to maintain a consistent distance of $2-3 \mathrm{~mm}$ between tissues and endoscopic camera lens (Figure 2). The microvascular 


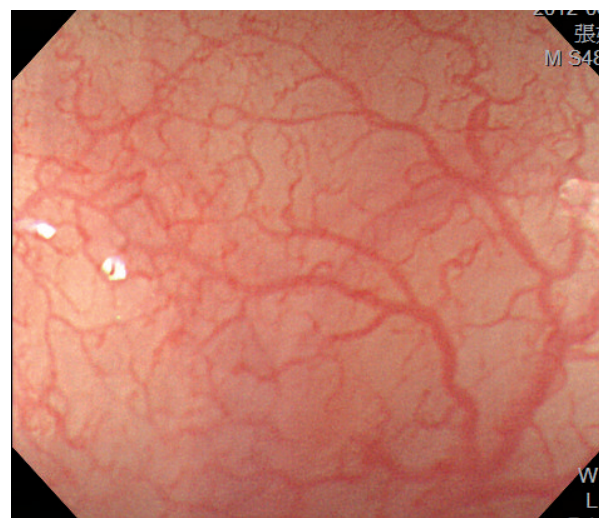

(a)

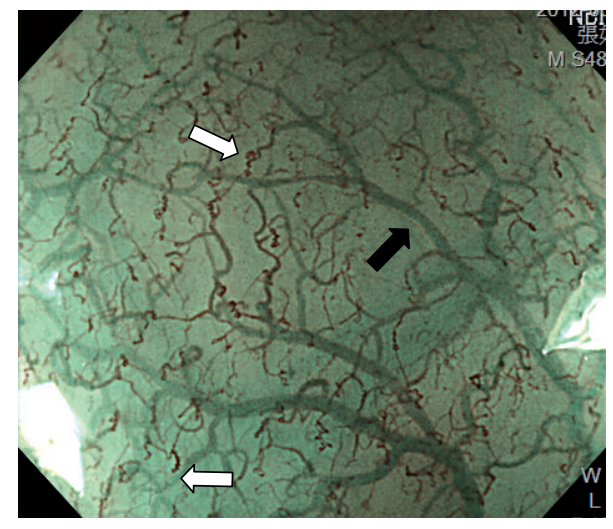

(b)

FIGURE 1: (a) Magnifying endoscopy under white-light imaging shows microvasculature of the normal esophagus. (b) Magnifying endoscopy under narrow-band imaging improves visualization of the reddish-brown superficial vessels (white arrow, Inoue's classification of intraepithelial papillary capillary loops type II) and the cyanic hue deeper vessels (black arrow).

architecture and the invasiveness of neoplasm could be well delineated by the ME-NBI $[22,24,25]$.

\subsubsection{Flexible Spectral Imaging Color Enhancement. Flexible} spectral imaging color enhancement (FICE), or optical band imaging, is a dyeless optical contrast technique based on spectral estimation technology. Ordinary endoscopic pictures are taken by charge-coupled device camera in a regular endoscopy and arithematically processed. Different from NBI system with fixed wavelengths, composite FICE images are generated on a computer which allows viewing of an image taken under light at any suitable wavelength setting for specific condition [26, 27]. Between wavelength $400 \mathrm{~nm}$ and $695 \mathrm{~nm}$ of visible light, 60 spectral images at $5 \mathrm{~nm}$ interval can be selected. The digital processing system can make switchover between ordinary image and FICE image faster than the NBI system which uses the optical filter. Using unlimited combinations of selected spectral transmittance with dedicated wavelengths, the FICE system is useful in discriminating among nonneoplastic and neoplastic lesions of the UGI tract [28].

2.1.3. Autofluorescence Imaging System. Autofluorescence imaging (AFI) system produces real-time images by the detection of changes in autofluorescence of malignant tissues. The AFI system can detect the differences of concentration or depth distribution of endogenous fluorophores, such as collagen, nicotinamide, adenine dinucleotide, flavin, and porphyrins, between normal and cancerous tissues $[29,30]$. The autofluorescence with longer wavelength is emitted by excitation short-wavelength blue light $(395-475 \mathrm{~nm})$. The intensity of autofluorescence, green $(550 \mathrm{~nm})$ and red $(610 \mathrm{~nm})$ reflectance images, was provided for pseudocoloring. An image processor makes autofluorescence images to green color, the green reflectance image to red color, and the red reflectance image to blue color, then AFI pseudocolored images were composited. The difference in autofluorescence emission between normal and dysplastic/cancerous mucosa is likely due to changes in nuclear/cytoplasmic (N/C) ratio and concentrations of collagen and hemoglobin. Normal mucosa emits brighter autofluorescence than cancerous parts, thus, the composite color appears greenish. Because hemoglobin absorbs both autofluorescence and green light $(550 \mathrm{~nm})$, vessels or inflammatory mucosa that contain more hemoglobin were displayed as bluish. Because autofluorescence is absorbed well by dysplastic/cancerous mucosa, tumor parts appear magenta in the AFI image [29].

\subsubsection{Confocal Laser Endomicroscopy and Endocytoscopy.} Confocal laser endomicroscopy (CLE) was developed to enable living cellular and microvascular structures visualized with magnification level up to 1,000-fold and to provide better spatial resolution than conventional fluorescence microscopy [31]. A low-powered blue laser light is emitted and focused onto a point of interest in a defined microscopic field. The emanating light from the observed point is focused to a pin hole which rejects out-of-focus light and avoids contamination by light scattering from different focal planes. After passing the pin hole, the fluorescent light projects to a photodetection device and in turn transforms into electronic signals. Because the illumination and detection systems are in the same focal plane, the endomicroscopy is termed "confocal." All detected transformed electronic signals from the illuminated spot are measured and computed. Exogenous fluorescence contrast agents, such as fluorescein, acriflavine, or cresyl violet, are needed either in systemic or topical application to generate CLE images. The most common contrast agents are topical spraying acriflavine hydrochloride ( $0.05 \%$ in saline) or intravenous fluorescein sodium $(5-10 \mathrm{~mL}$ of a $10 \%$ solution). Two kinds of CLE devices are commercialized available: integrated into an endoscope (eCLE) (Pentax, Tokyo, Japan) and as a stand-alone probe (pCLE) capable of passage through the accessory channel of most endoscopes (Cellvizio, Mauna Kea Technologies, Paris, France) [32]. 


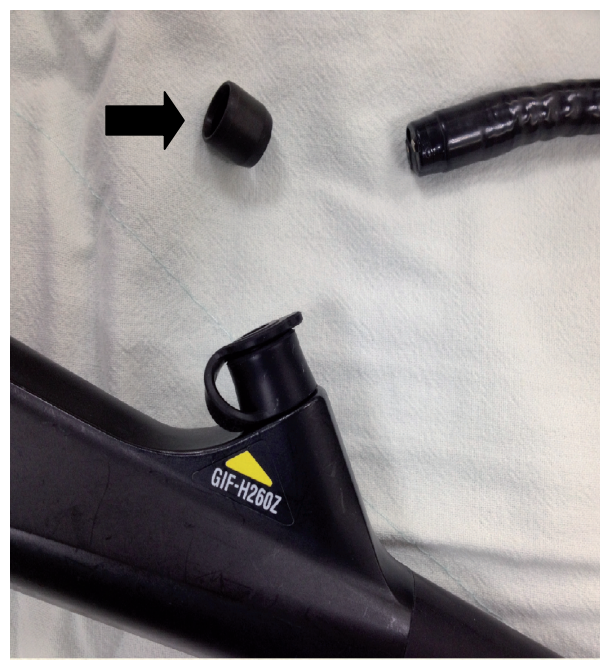

(a)

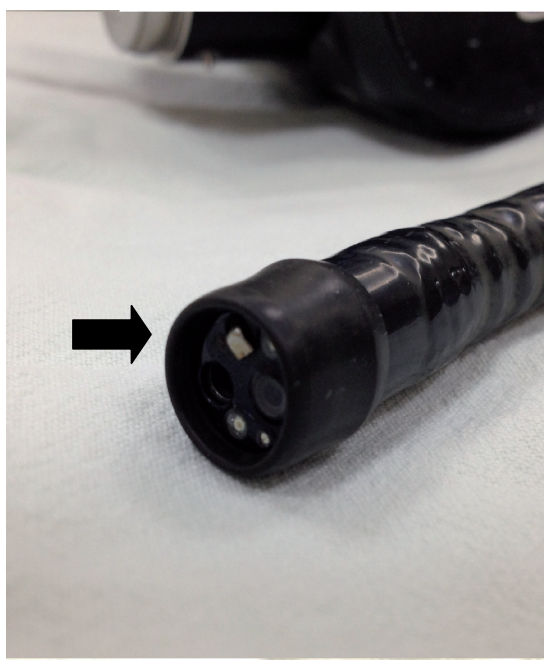

(b)

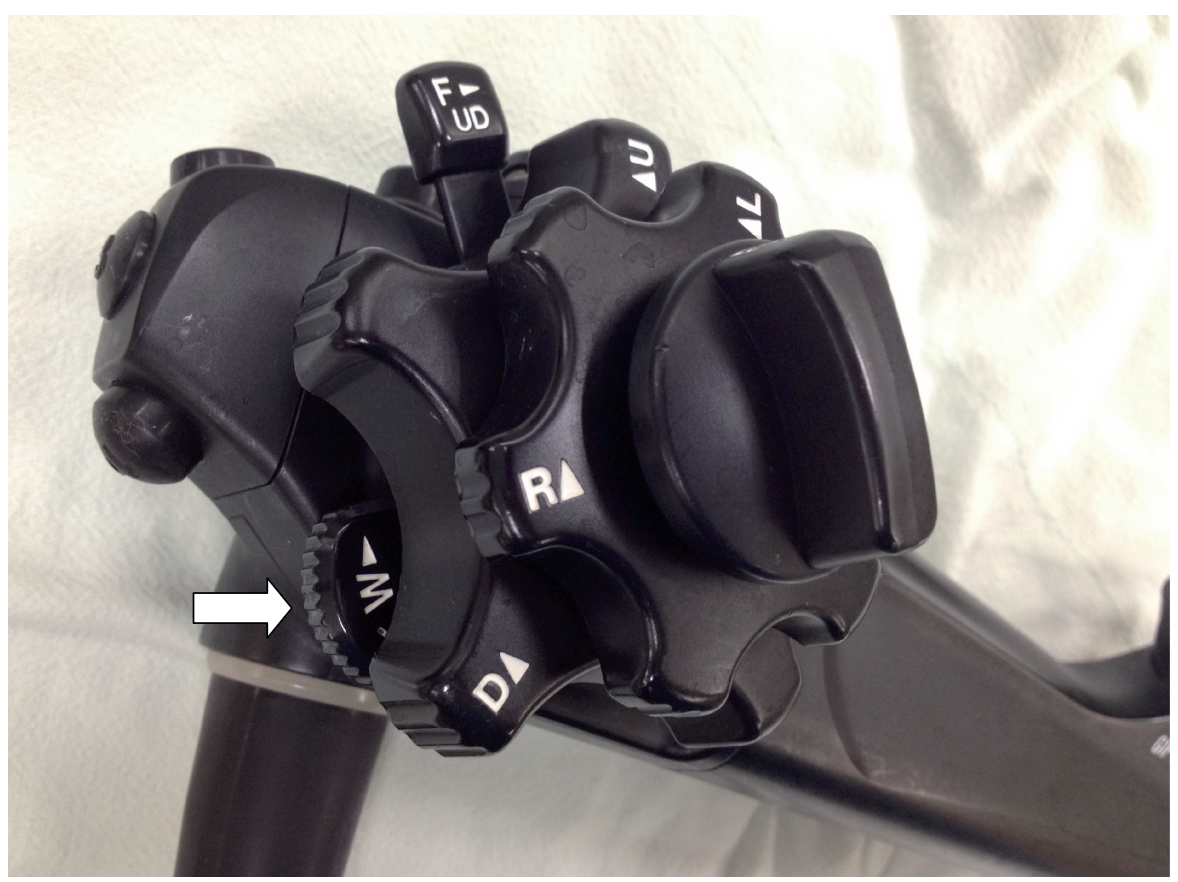

(c)

Figure 2: By adjusting a switchover apparatus (white arrow), the magnifying endoscope (GIF-H260Z, Olympus Medical Systems Corp, Tokyo, Japan) with plastic cap-fitted at its end (black arrow) can provide 80 -fold zooming images.

Endocytoscopy (EC) (Olympus, Tokyo, Japan) was developed using the principle of contact light microscopy with ultra-high magnification at 1,000- to 1,400-fold level [33-35]. In contrast to CLE which can visualize mucosal structures up to $250 \mu \mathrm{m}$ below the surface layer, EC only allows visualization of the very superficial $(50 \mu \mathrm{m})$ mucosal layer. After treating the mucosa with mucolytic agents, such as $\mathrm{N}$-acetylcysteine, mucosal staining with $1 \%$ methylene blue in the oesophagus and with $0.25 \%$ toluidine blue in the stomach and colon is sprayed for EC examination. About 60 seconds of exposure to the dye, repeat instillation of the mucosa is needed to remove excess dye before ultra-high magnification examination. The instruments of EC include probe-based (pEC) and endoscope-based (iEC) systems. The pEC system has 2 flexible catheter devices that provide ultra-high magnification imaging of the epithelial surface at 570 -fold or 1400 fold on a 19-inch monitor (or 450-fold and 1125-fold on a 14-inch monitor). The iEC system uses two separated lenses and is integrated into an 80-fold magnification endoscope enabling 450 - to 580 -fold magnification on a 19 -inch monitor $[33,35]$.

The observation at the level of virtual histology by the CLE and EC techniques offers real-time in vivo "optical or virtual" biopsy. These technological advances of endoscopy 
equipment might replace traditional endoscopy-guided biopsy which is sometimes insufficient to make correct diagnosis.

2.1.5. Optical Coherence Tomography. Optical coherence tomography (OCT) is a cross-sectional mapping of optical reflectivity by means of infrared light based on the principles similar to B-mode ultrasound [36]. The high resolution around $15 \sim 20 \mu \mathrm{m}$ makes acquisition of imaging nearly at the histology level possible. An optical probe with fiber optic and electrical cable in a flexible tube, and lateral scanner with lens at the distal end, is inserted into the working channel of endoscope with contact of target tissue. The targeted tissue is discriminated as layers determined by the time it takes for the infrared light to contact various layers of the intestinal wall and reflect back to the detector, optical interferometry [37]. The light is split into sampling and reference arms; the latter reflects from a mirror and returns to the same point where it originated then recombines with the sampling light. These two arms interfere, producing oscillations or fringes. By measuring the amplitude of oscillations and the coherence length, a function of depth and lateral coordinates can be computed to produce OCT images.

2.1.6. $i$-Scan. The i-scan technology utilizes the digital contrast method to produce high resolution enhanced images. Three kinds of image enhancement are available, that is, surface enhancement (SE), contrast enhancement (CE), and tone enhancement (TE) $[38,39]$. The SE mode enhances the light contrast by obtaining luminance intensity for each pixel, and edges of images are enhanced for more extensive observation of the glandular structures of mucosa surface. The CE mode adds blue light component and slightly suppresses the red and green components in lower luminance intensity area. As a result, the bluish-white discoloration of the lower luminance areas is produced for detailed observation of subtle irregularities of mucosal surface. In the TE mode, the RGB components of conventional endoscopic images are separated into each light component, and each isolated component is converted independently along the $\mathrm{S}$ or $\mathrm{J}$ tone curve by modification of input and output parameters, followed by reconstruction of each light component to produce TE images. The J type tone curve makes structural changes clearer by suppressing the maximal output of $\mathrm{R}$ component and enhancing overall $\mathrm{GB}$ component. The $\mathrm{S}$ type tone curve shifts the high $\mathrm{R}$ component area to a higher range or the low $\mathrm{R}$ component region to a further lower range of $\mathrm{R}$ to enhance the sensitivity to GB components. So far, about 6 types of TE are available for different tone enhancement for specific condition: TE-p (enhanced R component), TE-v (suppressed R component similar to NBI system), TE-b (for BE), TE-e (for esophagus), TE-g (for stomach), and TE-c (for intestines) [38]. The i-scan system can provide detailed observation of mucosal surface by digital contrast method with simple and quick pushingbutton switchover.

2.1.7. Chromoendoscopy. The most commonly used dyes for UGI tract mucosa are Lugol's solution, indigo carmine, acetic acid, and methylene blue [40]. Dyeing chromoendoscopy has been introduced over 40 years ago to enhance contrast differences between normal and neoplastic mucosa. For squamous epithelium of the esophagus, $0.5 \sim 5 \%$ Lugol's solution which is composed of iodine and potassium iodide can be well absorbed by glycogen-containing normal squamous epithelium showing brownish discolored "silk crape" like surface $[40,41]$. The dysplastic/cancerous squamous epithelium with less glycogen do not stain and appears white-yellowish Lugolvoiding areas. If a light-pink part (silver discoloration under NBI system) appears in the iodine-unstained region within 3 minutes after spraying, the lesion is highly suspected as HGIN (Figure 3) [42]. The pink-silver sign has the sensitivity and specificity of $91.9 \%$ and $94.0 \%$, respectively, to diagnose HGIN or invasive carcinoma [42]. However, certain side effects may develop after spraying Lugol's solution to esophagus, including chest pain, retrosternal cold sensation, nausea, or generalized itching allergic reactions [43]. To avoid unpleasant side effects, usually the concentration of $1.5-2 \%$ is sufficient to obtain adequate contrasting images. Sodium thiosulfate spraying after Lugol's chromoendoscopy can relieve irritation by Lugol's solution [43]. The 0.2 $0.4 \%$ indigo carmine solution can accentuate the border and surface topography by pooling into the crevices of the mucosal surface. The margin and mucosal pattern of the columnar epithelium of stomach and intestines can be well delineated by chromoendoscopy with indigo carmine solution. Using 1 3\% acetic acid for chromoendoscopy, "acetowhitening" reaction may develop due to reversible acetylation of nuclear proteins (Figure 4). This reaction only lasts a few minutes and is even more quickly lost in dysplastic/cancerous regions which become red faster than nondysplastic BE [44]. The methylene blue dye composed of methylthioninium chloride can be absorbed by absorptive epithelial cells of small intestine, colon, and intestinal metaplasia at any site of the gastrointestinal tract which are stained blue. By spraying 0.1 0.5\% methylene blue, specialized intestinal metaplasia (SIM) is stained as the presence of dark blue mucosa that persists despite vigorous irrigation, whereas staining pattern heterogeneity and decreased stain intensity suggest Barrett's high-grade dysplasia or cancerous changes. However, methylene blue chromoendoscopy should be cautiously performed because carcinogenesis risk could be increased due to oxidative damage to DNA by the photosensitized dye after whitelight exposure [45]. The clinical data supporting the potential carcinogenic effect of methylene blue is still inconclusive and needs further investigation [46].

\section{The Application of IEE in Esophagus}

\subsection{Narrow-Band Imaging System with Magnifying Endoscopy}

3.1.1. Squamous Cell Carcinoma of the Esophagus. Esophageal neoplasia can be detected as brownish discoloration areas by the NBI system because of its characteristic of hypervascularity $[22,24]$. By endoscopic screening with NBI system in high-risk population, the prevalence of advanced esophageal neoplasia could be as high as $28 \%$ [47, 48]. Even in head and neck cancer patients with trismus and difficulty in oral intubation of standard endoscopy, the use of ultrathin 


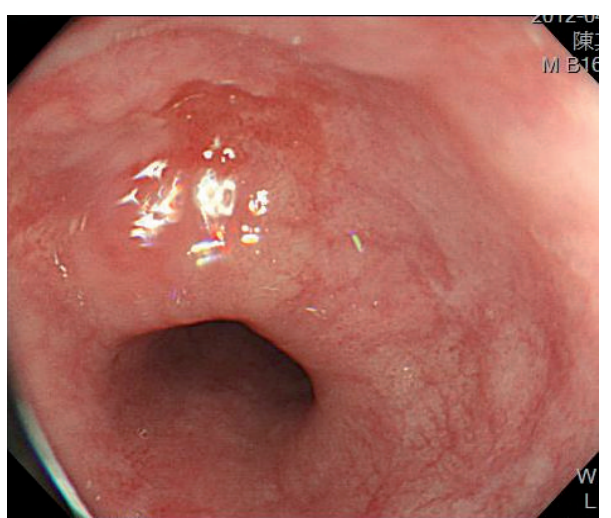

(a)

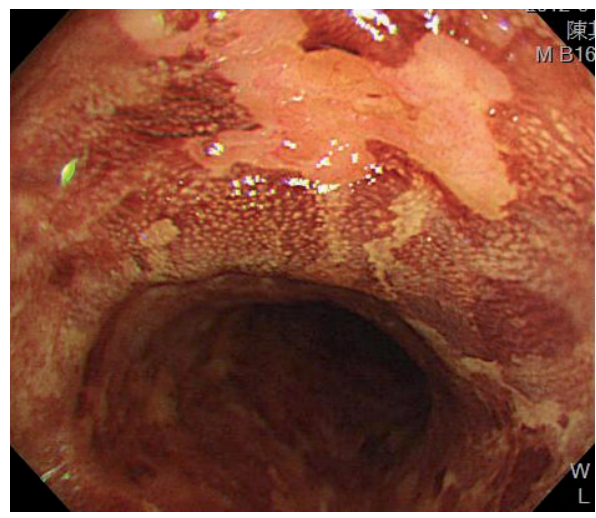

(c)

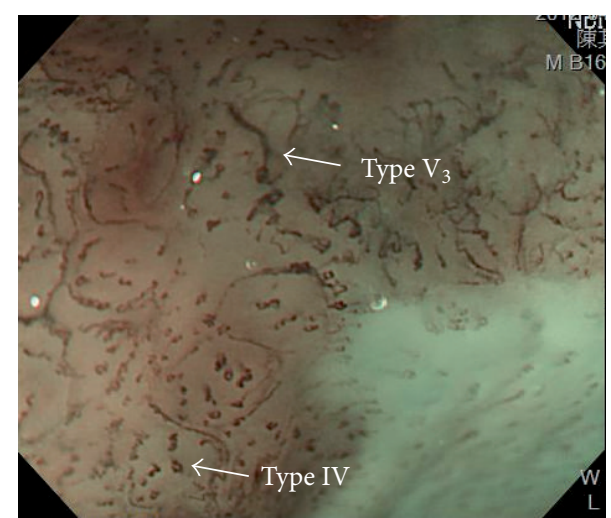

(b)

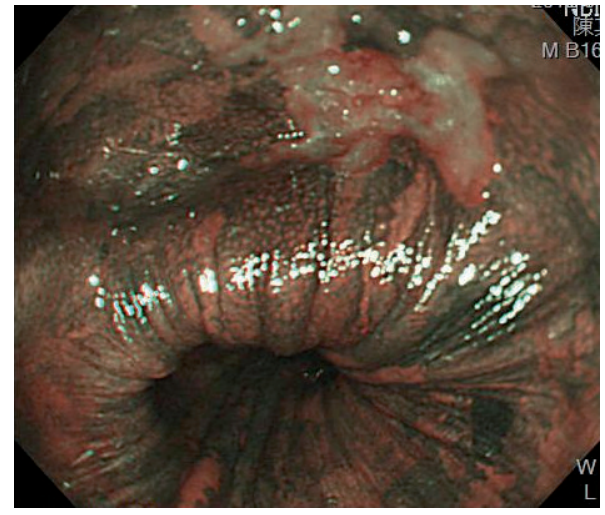

(d)

FIGURE 3: (a) Conventional white-light imaging endoscopy shows a type 0-IIc hyperemic lesion of esophagus. (b) Magnifying endoscopy with narrow-band imaging system reveals abnormal intraepithelial papillary capillary loops (Inoue's classification type $I V$ to $V_{3}$ ). (c) and (d) Chromoendoscopy with $1.5 \%$ Lugol's solution discloses Lugol-unstained area which appears pinkish under white-light imaging and silver pattern under narrow-band imaging 3 minutes after spraying dyes.

transnasal endoscope equipped with NBI system is feasible for esophagus screening (sensitivity $88.9 \%$ and specificity $97.2 \%$ for high-grade intraepithelial neoplasia (HGIN) and invasive carcinoma) [49]. By ME-NBI system observation of esophagus, the capillaries derived from the branching vessels in the submucosa extending to the epithelial layer appear as dark brownish tennis racket-shaped dots. These microvascular structures are named as "intraepithelial papillary capillary loops" (IPCLs) [25]. Because of angiogenesis in ESCC, the changes in morphology with four characteristics, including dilatation, tortuosity, meandering enlarged caliber changes, and variation in shape of IPCLs, are observed $[24,50,51]$. Brownish discoloration of background epithelium under ME-NBI was also significantly (OR 25.5, 95\% CI: 2.4-268) associated with mucosal high-grade dysplasia [52]. According to the different IPCLs pattern, ME-NBI can clearly distinguish esophageal neoplasia from nonneoplasia [51].

Inoue et al. further classified IPCLs into type I to $\mathrm{V}$ (Figure 5) [50, 53]. Type I IPCLs are observed in normal squamous epithelium. Type II IPCLs have elongation and/or dilatation changes and are often seen in esophagitis (Figure 1). Type III IPCLs which are iodine voiding under Lugol's solution staining have minimal color changes from type I
IPCLs without proliferation, and these changes correspond to chronic esophagitis or low-grade intraepithelial neoplasia (LGIN). Type IV IPCLs have two to three of four morphology changes and are associated with HGIN or carcinoma in situ. Type V IPCLs demonstrate all four morphology changes. Type V IPCLs are further categorized from type V-1, V-2, $\mathrm{V}-3$ to $\mathrm{V}_{N}$. In type V-1 IPCLs representing carcinoma in situ (m1), changes include dilatation with tortuosity, meandering irregular calibers, and variable forms (Figure 6). Type V-2 IPCLs which are found in cancer invading lamina propria (m2) are the extension form of V-1. Type V-3 IPCLs corresponding to cancer involving the muscularis mucosa $(\mathrm{m} 3)$ or superficial submucosa ( $\mathrm{sml}$ ) are advanced destruction form of capillaries running in a horizontal plane (Figure 6). As for the type $V_{N}$ IPCLs (Figure 7), generation of large-caliber new tumor vessels which could be 10 times larger than the V-3 IPCLs appears in deeper submucosal cancer (sm2). The accuracy of depth prediction by Inoue's classification of IPCLs was about $83.3 \%$ [54].

Another classification system of IPCLs was proposed by Arima et al. [55]. IPCLs are categorized from type 1 to 4 . Type 1 was characterized by thin, linear capillaries in the subepithelial papilla. Type 2 appears as regularly arranged vessels with 


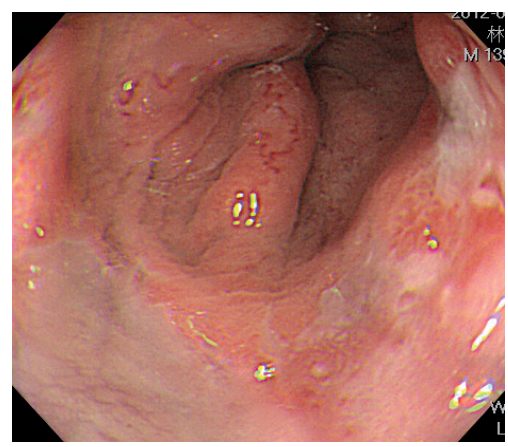

(a)

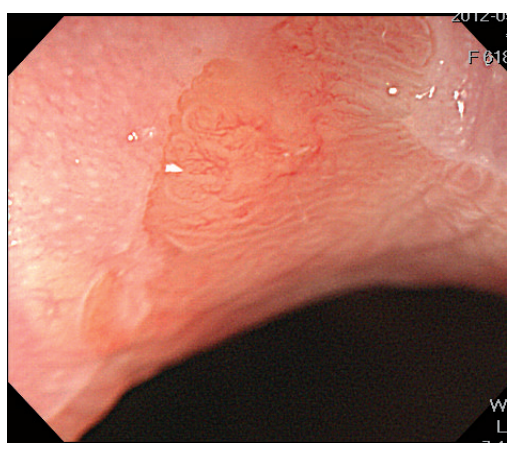

(d)

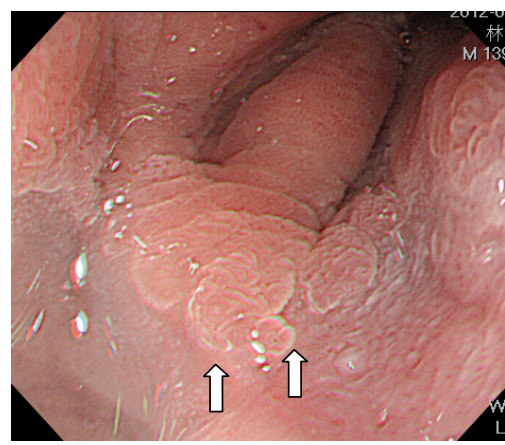

(b)

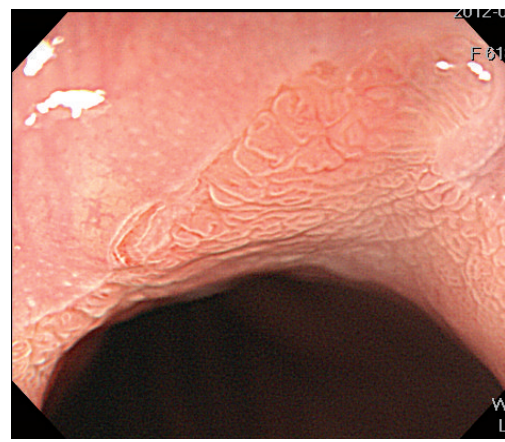

(e)

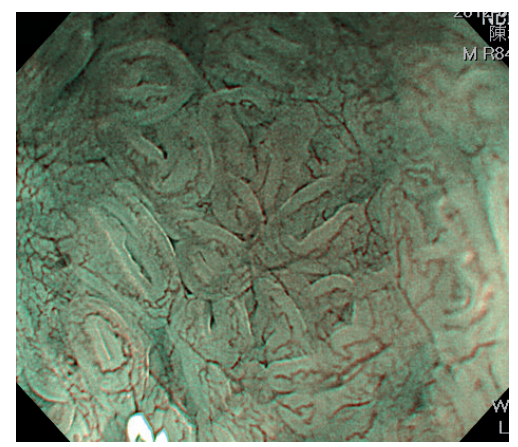

(c)

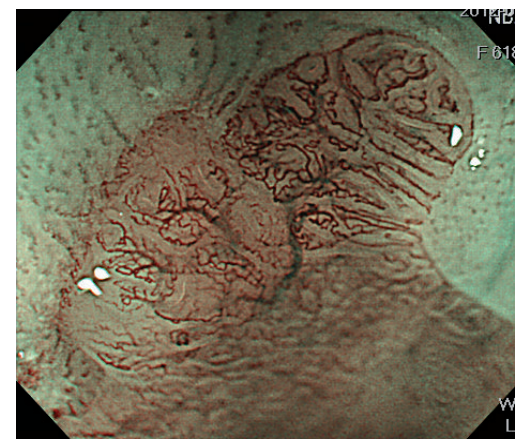

(f)

Figure 4: (a) White-light imaging endoscopy shows Barrett's esophagus. (b) Chromoendoscopy with 2\% acetic acid shows "acetowhitening" reaction of the mucosa with intestinal metaplasia. (c) Magnifying endoscopy with narrow-band imaging system reveals nondysplastic mucosa presenting cerebriform or gyri-like pit pattern with superficial blood vessels regularly situated between the mucosal ridges. (d) Magnifying endoscopy with white-light imaging shows increased vascularity of mucosa breaks which appears as a villous pit pattern (e) after acetic acid spraying. (f) Magnifying endoscopy under narrow-band imaging system disclosed low-grade dysplasia with irregular/disrupted mucosal patterns and irregular vascular patterns.

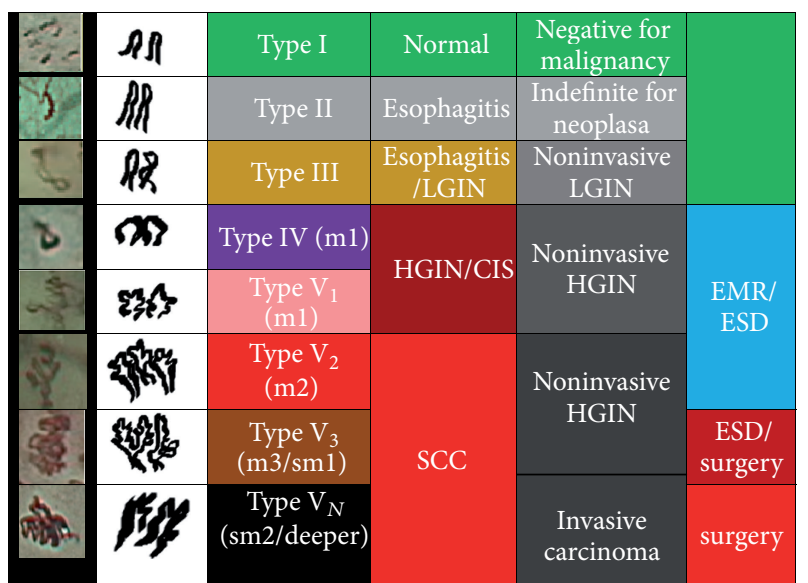

FIGURE 5: Inoue's classification of intraepithelial papillary capillary loops for esophageal neoplasia.

dilatation and variable branching or spiral enlargement. Type 3 was characterized by destruction of crushed vessels with an irregular caliber. Type 4 was characterized by irregular multilayered, irregularly branched, or reticular vessels. According to Arima's classification system, most were normal mucosa (79.5) or inflammatory changes (15.4\%) in type $1 ; 64.1 \%$ were inflammation and $14.1 \%$ were mild-to-moderate dysplasia in type $2 ; 86.9 \%$ were $\mathrm{ml} \sim \mathrm{ml}$ cancers in type 3 ; and $89.6 \%$ were $\mathrm{m} 3$ or deeper cancers in type 4 . Moreover, the size of the avascular areas (AVAs) was closely associated with the cancer invading depth. Examination of the size of AVAs as well as the presence of stretched type 4 IPCLs can predict the extent and depth of HGIN or cancer well with correction rate up to $94.2 \%$ [55].

Identifing the depth of invasion and histology for superficial esophageal neoplasia is important for treatment strategy. Close periodically endoscopic surveillance is mandatory for LGIN of esophagus, and endoscopic resection should be done for HGIN of esophagus $[8,9,16,17,24]$. Given the low risk of LN metastasis for mucosal cancers of esophagus, ESD or EMR should be done for $\mathrm{m} 1 / \mathrm{m} 2$ cancers with absolute indication and relatively applied to $\mathrm{m} 3$ cancer if en bloc resection is possible without evidence of lymphovascular invasion. To provide rapid real-time information of predicted histology and invasiveness of neoplasia, NBI system, especially in combination with $\mathrm{ME}$, should be used in routine surveillance of esophageal cancer $[8,9,24,47,51]$.

3.1.2. Premalignant Lesions of Adenocarcinoma-Barrett's Esophagus. High resolution magnifying endoscopy combined with NBI system can improve the detection of SIM 


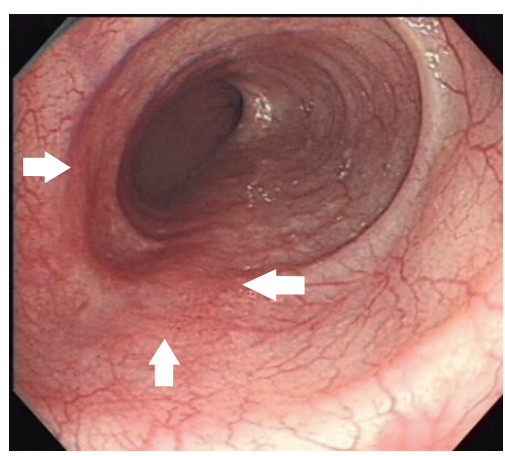

(a)

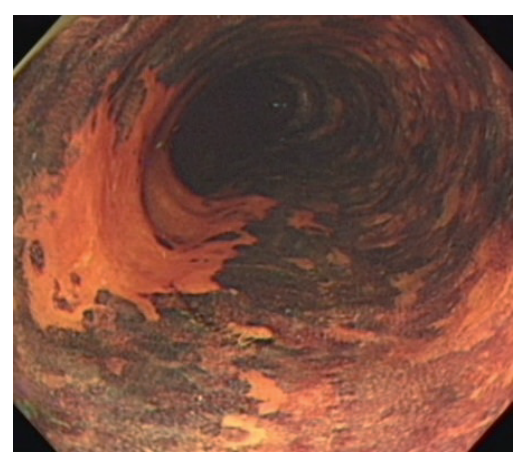

(b)

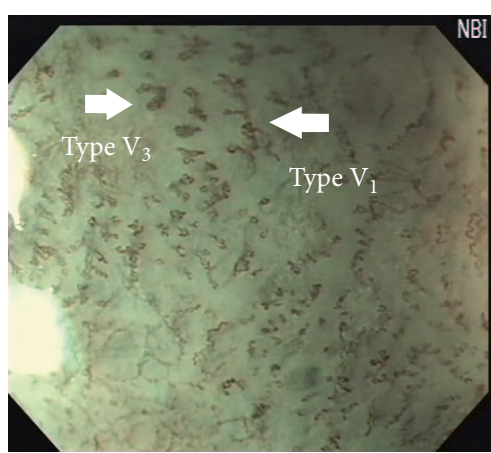

(c)

FIGURE 6: (a) White-light imaging endoscopy shows a type 0-IIb lesion with mildly hyperemic change of mucosal surface. (b) Chromoendoscopy after spraying 1.5\% Lugol's solution discloses Lugol-unstained appearance. (c) Magnifying endoscopy with narrow-band imaging system reveals abnormal superficial vessels (Inoue's classification of intraepithelial papillary capillary loops type V).

and dysplasia of distal esophagus. By thorough examination of microstructure and microvascular patterns, the sensitivity, specificity, positive predictive value (PPV), and negative predictive value (NPV) of the combination of regular microstructural pattern (tubular/villous/linear) and absent microstructural pattern to detect SIM were $100 \%$, $78.8 \%, 93.5 \%$, and $100 \%$, respectively [56]. The sensitivity, specificity, PPV, and NPV of the irregular microvascular/microstructural pattern for the prediction of HGIN were $90 \%, 100 \%, 99.2 \%$, and 100\%, respectively [56]. Singh et al. has developed a simplified grading system for BE by using ME-NBI examination [57]. The PPV and NPV for lesions with type $\mathrm{A}$ (round pits and regular microvasculature) for the histology of columnar mucosa without SIM were $100 \%$ and $97 \%$, respectively; for type B (villous/ridge pits with regular microvasculature) or type $\mathrm{C}$ (absent pits with regular microvasculature) for histology of SIM, they were $88 \%$ and $91 \%$, respectively and for type D (distorted pits with irregular microvasculature) for histology of HGIN $81 \%$ and 99\%, respectively [57]. A recent meta-analysis has shown good diagnostic performance of ME-NBI system for BE [58]. For diagnosing HGIN, the pooled sensitivity, specificity, diagnostic OR, and area under the curve (AUC) were $96 \%$, 94\%, 342.49 (95\% CI 40.49-2896.89), and 0.99, and for the characterization of SIM, the pooled sensitivity, specificity, diagnostic OR, and AUC were 95\%, 65\%, 37.53 (95\% CI $6.50-217.62$ ), and 0.88 [58]. By targeted biopsy of suspicious lesions identified by ME-NBI system, the number of random biopsies may be reduced and the diagnostic yield may be improved.

\subsection{Flexible Spectral Imaging Color Enhancement}

3.2.1. Squamous Cell Carcinoma of the Esophagus. There are limited experiences in the application of FICE on the surveillance for ESCC. The method of selecting a suitable combination of wavelengths of RGB lights for esophageal squamous epithelium has not been well established. Inoue et al. have shown that the specific FICE modes A (R $550 \mathrm{~nm}$, gain 2; G $500 \mathrm{~nm}$, gain 2; B $470 \mathrm{~nm}$, gain 3) and C (R $540 \mathrm{~nm}$, gain 2; G $415 \mathrm{~nm}$, gain 2; B $415 \mathrm{~nm}$, gain 3) significantly enhanced the visibility of IPCLs of ESCC mucosa [27]. Some investigators have demonstrated a superior outcome in the diagnostic yield of esophageal capsule endoscopy by combination of the FICE system mode A with the PillCam ESO2 (Given Imaging, Yoqneam, Israel) [59]. However, although the microvessels could be visible more clearly by FICE system than conventional white-light imaging system, the relatively lower resolution of FICE than that of NBI system hampers further categorization of the microvascular morphology.

\subsubsection{Premalignant Lesions of Adenocarcinoma-Barrett's} Esophagus. The identification of the palisading vessels of the esophagus and the termination of gastric folds is important to endoscopic diagnosis of BE. FICE system can improve visualization of the end of palisade vessels of the esophagus. Osawa et al. has demonstrated that the FICE system enables clear visualization of the demarcation of BE mucosa, gastric folds, and esophageal palisade vessels [60]. Pohl et al. has found that the FICE system has comparable diagnostic performance to acetic acid chromoendoscopy, with the sensitivity of targeted biopsy for HGIN/early cancer of $87 \%$ [61].

\subsection{Autofluorescence Imaging System}

3.3.1. Squamous Cell Carcinoma of the Esophagus. AFI has higher accuracy for diagnosing early ESCC than WLI system, especially for flat/elevated lesions or those with diameter $\geqq 20 \mathrm{~mm}$, but ulcerations or inflammatory changes and depressed lesions or those with diameter $<20 \mathrm{~mm}$ may cause misdiagnosis by AFI evaluation [29, 62]. Recently, a phase-II study in Japan has shown unsatisfactory diagnostic power of AFI system in screening esophageal HGINs, with sensitivity of $71 \%$ (95\% CI 55-87\%) and PPV of 25\% (95\% CI 16-34\%), especially for lesions $\leqq 10 \mathrm{~mm}$ [63]. Therefore, AFI system plays a limited role in screening ESCC, especially for those with small size and depressed morphology.

3.3.2. Premalignant Lesions of Adenocarcinoma-Barrett's Esophagus. Panjehpour et al. has conducted one of the earliest studies using laser-induced AFI system to detect HGIN 


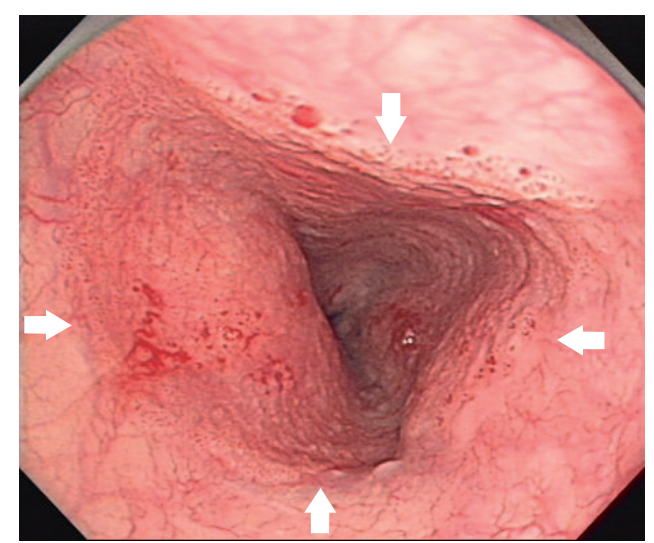

(a)

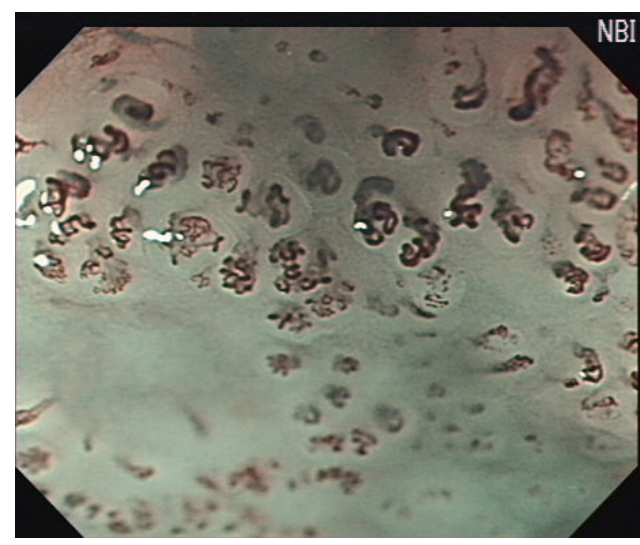

(c)

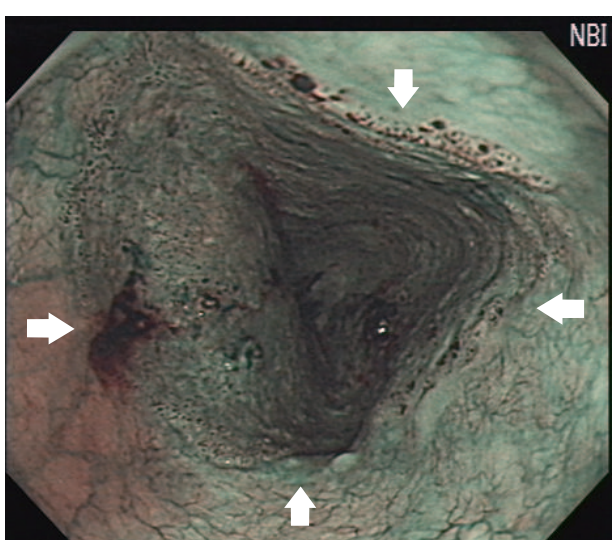

(b)

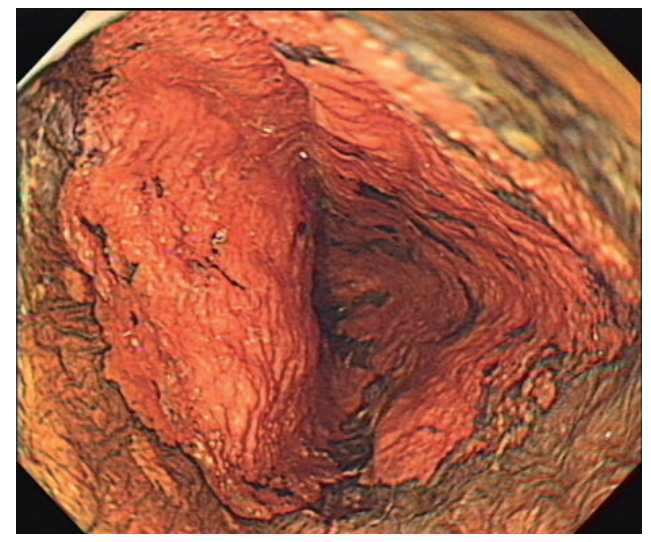

(d)

Figure 7: (a) Conventional endoscopy shows an esophageal circumferential long-segment neoplasia with hyperemic changes and nodularity of surface mucosa which turns to brownish discoloration (b) under narrow-band imaging system. (c) Under magnification with narrow-band imaging, abnormal superficial vessels are well demonstrated (Inoue's classification type $\mathrm{V}_{N}$ ). (d) Chromoendoscopy with $1.5 \%$ Lugol's solution shows extended Lugol-voiding area.

in BE mucosa [64]. The analysis of the fluorescence spectra using the differential normalized fluorescence intensity at $480 \mathrm{~nm}$ index showed that $96 \%$ of nondysplastic BE samples were classified as benign, $100 \%$ LGIN samples as benign, $90 \%$ of HGIN samples as premalignant, and $28 \%$ of low-grade with focal high-grade dysplasia samples as premalignant. Differences in 5-aminolevulinic acid (10 mg/kg orally 3 hours before endoscopy) induced protoporphyrin IX fluorescence intensity at $635 \mathrm{~nm}$ can also be used to distinguish dysplastic to nondysplastic lesions with the sensitivity of $77 \%$ and specificity of $71 \%$ [65]. Although the AFI has high sensitivity and NPV, its strength is limited by the moderate specificity and PPV for detection of dysplasia in BE lesions [66]. Using NBI system as an adjunct to increase accuracy of detecting dysplasia, the false positive rate of AFI system can be reduced from $40 \sim 81 \%$ to $10 \sim 48 \%$ [66-68]. Thereby, endoscopic trimodal imaging (ETMI) system incorporating high-resolution endoscopy, AFI, and NBI systems has been developed to increase detection of HGIN of BE [68]. However, recent randomized multicenter crossover studies have shown that the yield of targeted biopsies of ETMI was significantly inferior to the overall yield of standard video endoscopy with 4-quadrant random biopsies every $2 \mathrm{~cm}[67$, 69]. Moreover, the interobserver agreement for AFI suspected lesions is only fair to substantial $(\kappa=0.62$ for experts and $\kappa=0.28$ for nonexperts). At present, ETMI seemingly cannot replace random biopsies by standard video endoscopy for detection of dysplastic lesions.

\subsection{Confocal Laser Endomicroscopy and Endocytoscopy}

3.4.1. Squamous Cell Carcinoma of the Esophagus. CLE can be used in differentiating neoplastic lesions from normal epithelium. IPCLs could be demonstrated by CLE (Pentax EC-3870 CIFK, Pentax, Tokyo, Japan). The superficial ESCC has higher proportion of irregular arrangement of epithelial cells. (79.4\% versus $10.0 \%, P<0.001)$, increased diameter of IPCLs $(26.0 \mu \mathrm{m}$ versus $19.2 \mu \mathrm{m}, P<0.001)$, and irregular shape IPCLs $(82.4 \%$ versus $36.7 \%, P=0.0002)$ than normal mucosa by CLE examination [70]. By the defined criteria for cellular (dark cells with different sizes and irregular architecture, without clearly visible borders) and vascular (twisted and irregular vessels, elongated capillaries with leakage of fluorescein) changes, CLE has an overall accuracy of $95 \%$ and 
the sensitivity and specificity of $100 \%$ and $87 \%$, respectively, for diagnosing early ESCC [71]. However, the proportion of images with good quality was still unsatisfactory (less than $40 \%$ ), and the interobserver agreement was substantial $[70,71]$.

EC observation could potentially replace the role of histologic examination on biopsied specimens in the diagnosis of ESCC. Inoue et al. used iEC type XGIF-Q260EC1 (Olympus Medical Systems Corp. Tokyo, Japan) which provides 450 -fold magnification power and observation area about $400 \times 400 \mu \mathrm{m}^{2}$ to in vivo evaluate tissue atypia of the esophagus [72]. Endocytoscopic atypical (ECA) was classified into five grades: ECA 1- large, cytoplasm-rich regularly arranged cells with a rhomboid shape (normal); ECA 2- round cells with different-sized small nuclei (inflammatory changes); ECA 3- small size but the nuclei are still compact (borderline lesions); ECA 4- higher cell density with an increased N/C ratio (suggestive of malignant lesions); ECA 5- irregularly arranged cells with various sizes with a high $\mathrm{N} / \mathrm{V}$ ratio (definitely malignant lesions) [72]. By this grading system, the overall accuracy to differentiate between nonmalignant and malignant issues was $82 \%$. Kumagai et al. further categorized the EC findings into Type 0 to 3: Type 0 - normally stained with iodine solution; Type 1- unstained with iodine, but showing normal squamous epithelial cells (a low cell density with a low N/C ratio without nuclear abnormality); Type 2unstained with iodine, showing a high cell density but no evident nuclear abnormality; Type 3- unstained with iodine, but with evidently increased nuclear density and abnormality [73]. There were high degrees of agreement for Type 1 (90.9\% with normal or inflammatory changes) and Type $3(92.3 \%$ with HGIN or invasive carcinoma) lesions with the histologic diagnosis, especially using the pEC type XEC120U (Olympus Medical Systems Corp. Tokyo, Japan) which provides 1125fold magnification and a tissue field of view measuring $120 \times$ $120 \mu \mathrm{m}^{2}$ [73].

CLE and EC are promising in vivo optical biopsy tools; however, overcoming technical problems concerning sufficient image quality and universal criteria for optical histologic diagnosis is essential before popular clinical application $[31,74]$.

3.4.2. Premalignant Lesions of Adenocarcinoma-Barrett's Esophagus. CLE can be used to distinguish between different types of epithelial cells and delineate cellular and microvascular changes in BE epithelium. Earlier study conducted by Kiesslich et al. has shown that BE and associated neoplasia can be predicted by CLE with a sensitivity of $98.1 \%$ and $92.9 \%$ and a specificity of $94.1 \%$ and $98.4 \%$, respectively (accuracy, 96.8\% and 97.4\%) [75]. A prospective randomized, double-blinded, controlled crossover trial disclosed that CLE with targeted biopsy doubled the diagnostic yield for neoplasia and was equivalent to the standard 4-quadrant biopsy procedure for the diagnosis of neoplasia, and nearly twothirds of patients did not need any mucosal biopsies to make final diagnosis [76]. Another large prospective international multicenter study has demonstrated that pCLE combined with high-definition (HD) WLI significantly improved the ability to detect neoplasia in BE patients compared to $\mathrm{HD}$ WLI alone (sensitivity $34.2 \%$ versus $68.3 \%, P=0.002$ ) [77]. A consensus for standardization of pCLE image criteria, called "Miami classification", has been introduced to describe diagnostic parameters to differentiate between normal squamous epithelium, nondysplastic BE, HGIN, and adenocarcinoma in BE [78]. However, some studies showed that poor positive predictive value $(46 \sim 67 \%)$ for pCLE to evaluate neoplasia in $\mathrm{BE}$ and pCLE can only be possibly regarded as noninferior to standard endoscopic biopsy $[79,80]$. Although emerging evidence supports the benefits of CLE application in BE, more data are needed to justify this novel approach in the clinical setting.

Pohl et al. has assessed the accuracy of EC in correlation with histology to distinguish neoplasia from $\mathrm{BE}$ in premarked areas [81]. In this study, only $23 \%$ of images with lower magnification (450-fold) were interpretable to identify characteristics of neoplasia and $41 \%$ with higher magnification (1125-fold). PPV and NPV for HGIN or cancer were 0.29 and 0.87 , respectively, for 450 -fold magnification and 0.44 and 0.83 , respectively, for 1125 -fold magnification. Endoscopic histology using EC lacks sufficient image quality to currently assist in identifying neoplastic areas in BE, and there are needs for an initial macroscopic wide-field surveillance technique to identify suspicious areas.

\subsection{Optical Coherence Tomography}

3.5.1. Squamous Cell Carcinoma of the Esophagus. OCT is not used for screening but can provide information for $\mathrm{T}$ staging of ESCC. Hatta et al. have demonstrated using OCT for preoperative staging with high degree of overall accuracy (92.7\%) (m1/m2, 94.9\%; m3, 85.0\%; sm, 90.9\%) [82]. Moreover, a nonrandomized comparative study has shown higher accuracy for $\mathrm{m} 1 / \mathrm{m} 2$ cancer staging by using OCT than that by using EUS with $20-\mathrm{MHz}$ miniature probe $(94.6 \%$ versus $80.6 \%, P<0.05)$ [83]. However, because of limited depth of penetration by OCT, the deeper submucosa and structures beyond the muscularis propria cannot be welldelineated. Further randomized prospective studies are needed to demonstrate the role of OCT in staging of superficial ESCC.

\subsubsection{Premalignant Lesions of Adenocarcinoma-Barrett's} Esophagus. OCT is a promising diagnostic tool for optical biopsy, and it can reduce the need for random biopsies with standard WLI endoscopy. Poneros et al. has defined OCT images for SIM with sensitivity and specificity of $97 \%$ and $92 \%$, respectively, by the characteristics of (1) absence of the layered structure of normal squamous epithelium and the vertical "pit and crypt" morphology of gastric columnar epithelium, (2) distorted architecture with heterogeneous tissue contrast and an irregular surface, and (3) presence of submucosal glands [84]. A prospective double-blinded study has an accuracy of $78 \%$ for the detection of dysplasia in BE mucosa [85]. Using computer-aided diagnosis system for classification of BE mucosa by OCT imaging may yield 


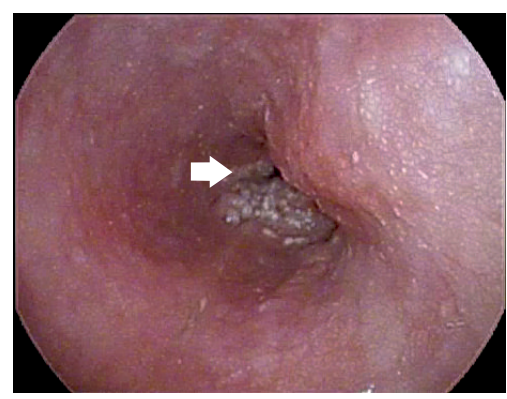

(a)

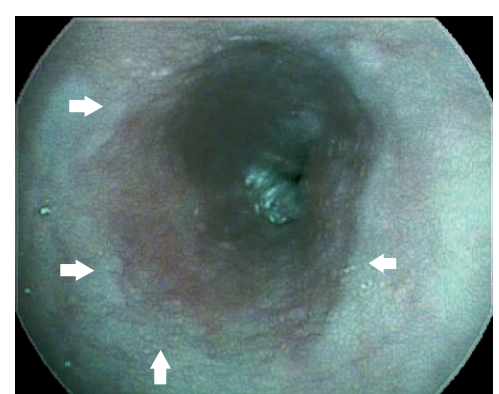

(b)

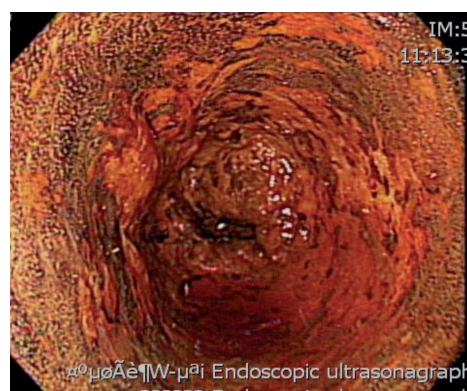

(c)

FIGURE 8: (a) Conventional white-light imaging endoscopy shows an ulcerative mass with lumen obstruction of the esophagus. (b) i-scan (SE 6+, CE 4+, TE-e) discloses reddish discoloration of the adjacent mucosa which is Lugol unstained (c), and the pathology is high-grade intraepithelial neoplasia.

higher accuracy of $84 \%$ for detection of dysplasia [86]. Moreover, the application of 3D-OCT imaging can provide realtime information for endoscopic ablation therapy response and identify residual dysplastic lesions which need further ablation [87]. OCT has the potential for diagnosing BE and differentiating dysplastic from nondysplastic lesions without histological biopsy.

3.6. $i$-Scan. There are limited data on the application of i-scan for screening of esophageal precancerous or cancerous lesions although premalignant mucosa of esophagus can be well delineated by i-Scan (Figure 8). However, i-scan can efficiently detect minimal changes of mucosa in patients with gastroesophageal reflux disease [88, 89].

\subsection{Chromoendoscopy}

3.7.1. Squamous Cell Carcinoma of the Esophagus. By using chromoendoscopy with Lugol's solution for screening ESCC in high-risk population, such as alcoholics or head and neck cancer patients, the prevalence of HGIN or invasive carcinoma of the esophagus could range from $3.2 \%$ to $16.6 \%$ [47]. As long as the concentrations of cellular glycogen content change, differences in degree of iodine staining enhance the contrast of the abnormal squamous epithelium. However, because lesions with chronic inflammation, squamous hyperplasia, or LGIN could be iodine unstained, Dawsey et al. has found that the chromoendoscopy with Lugol's solution has high sensitivity (96\%) but low specificity (63\%) for identifying HGIN or invasive carcinoma of esophagus [90]. Lee et al. has also demonstrated lower diagnostic performance of chromoendoscopy with Lugol's solution (sensitivity $88.9 \%$, specificity $72.2 \%$ ) than that of NBI system (sensitivity $88.9 \%$, specificity 97.2\%) in ESCC screening [49]. Nevertheless, to well delineate the spread of superficial esophageal neoplasia, especially for flat or slightly depressed lesions, iodine staining by spraying Lugol's solution before EMR or ESD is essential to mucosa marking of endoscopic resection margin.

3.7.2. Premalignant Lesions of Adenocarcinoma-Barrett's Esophagus. The dyes mainly applied to the survey of $\mathrm{BE}$ are methylene blue, acetic acid, and indigo carmine. The sensitivity, specificity, and overall accuracy of methylene blue for detecting SIM is $98 \%, 61 \%$, and 95\%, respectively [91, 92]. Endo et al. categorized BE pit patterns by ME-WLI into five types: small round, straight, long oval, tubular, and villous pit patterns [93]. The tubular and villous pit patterns which are positive staining for methylene blue are closely associated with the presence of SIM. Although a previous randomized crossover study has shown superior diagnostic accuracy (75\% versus $68 \%, P=0.032)$ of the methylene blue directed biopsy technique to that of the random biopsy technique for identifying SIM [94], a recent meta-analysis has conflicting result [95]. The technique of methylene blue chromoendoscopy has only comparable yield with random biopsy for the detection of SIM and dysplasia [95].

A significant improvement in detecting SIM of esophagus by acetic acid chromoendoscopy has been documented. Based on the acetowhitening reaction of columnar epithelium, chromoendoscopy with acetic acid can enhance the architecture of BE epithelium. The whitening reaction is lost in dysplastic tissues earlier than nondysplastic mucosa, aiding further distinguishing neoplasia from normal epithelium. A prospective randomized crossover study has shown that acetic acid-guided biopsies with the adjunct of ME were superior to standard video endoscopy with random biopsies (78\% versus $57 \%$ ), and the number needed to confirm BE was reduced [96]. ME with acetic-acid staining had an accuracy of $83.8 \%$ for prediction of BE [96]. Even without magnifying, acetic-acid chromoendoscopy can identify dysplasia or cancer in BE with the sensitivity and specificity of $95.5-100 \%$ and $80-97.7 \%$, respectively $[97,98]$.

\section{The Application of IEE in Stomach}

\subsection{Narrow-Band Imaging System with Magnifying Endoscopy}

4.1.1. Helicobacter pylori-Associated Gastritis. NBI alone [99], HD-ME [100], and ME-NBI [101] all had good correlation between histopathological findings and $\mathrm{Hp}$-associated gastritis, atrophic gastritis, and IM. HD-ME can reliably identify the normal gastric mucosa, Hp-associated gastritis, and gastric atrophy [100]. By identifying changes in morphology of subepithelial capillary network (SECN), connecting 


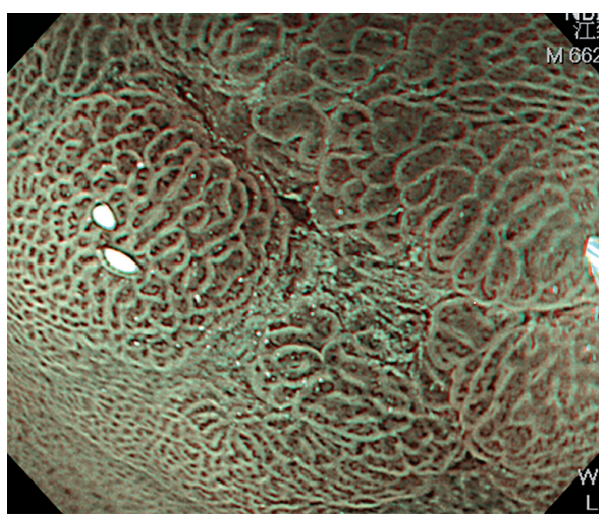

(a)

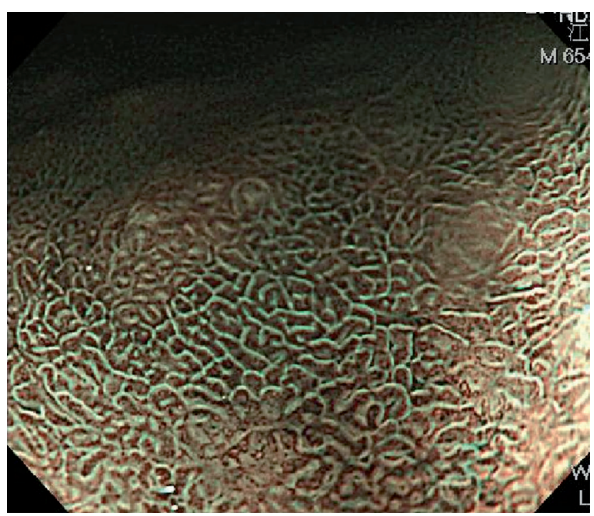

(b)

FIGURE 9: Magnifying endoscopy with narrow-band imaging of intestinal metaplasia change of gastric mucosa shows light blue crest sign (a fine, blue-white line on the crests of the epithelial surface).

venules and mucosal pits, the sensitivity, specificity, PPV, and NPV for predicting an Hp-infected stomach and gastric atrophy were $100 \%$ (95\% CI 83.9-100\%), 90\% (95\% CI 66.898.2\%), 92.7\% (95\% CI 93.2-97.3\%), 96\% (95\% CI 87.998.9\%), and $83.8 \%$ (95\% CI 65.5-93.9\%), 85.7\% (95\% CI 62.6-96.2\%), 100\% (95\% CI 92.9-100\%), and 97.3\% (95\% CI 89.6-99.5\%), respectively [100]. The magnified views of Hprelated gastritis significantly differed from normal mucosa presenting collecting venules and true capillaries forming a network with gastric pits in the center [102]. With the adjunct of NBI system to HD-ME, the mucosal pit pattern and microvascular architecture could be visualized more clearly. $\mathrm{Hp}$-infected stomach and the histological severity of gastritis and atrophy can be predicted accurately [101].

4.1.2. Gastric Intestinal Metaplasia. Gastric IM is a risk factor of intestinal-type gastric cancer, but WLI was not adequate to detect IM of stomach [103]. NBI system with and without magnification can excellently diagnose GIM with good histological agreement. Uedo et al. used a light blue crest (LBC) on epithelial surface by ME-NBI as a maker to diagnose IM with the sensitivity and specificity of $89 \%$ and 93\%, respectively [104]. The LBC is a fine, blue-white line on the crests of the epithelial surface (Figure 9), and it can only be detected under wavelengths of 400-430 nm. A multicenter study showed that regular ridge or tubulovillous mucosa can accurately predict IM with the sensitivity, specificity, and accuracy of $89 \%, 90 \%$, and $90 \%$, respectively [105].

4.1.3. Early Gastric Cancer. Conventional endoscopy has the limitation in detecting lesions by morphological changes, and the diagnosis of malignancy depends on pathology. Few morphologic changes could be detected by WLI to differentiate malignant from nonmalignant lesions in EGC. The minute surface structure and microvessels observed by ME were related to histopathological findings [106]. Combining NBI system with ME could maximize the benefit for diagnostic accuracy, such as microvascular architecture, microsurface structure, and demarcation line (DL) between cancer and surrounding mucosa $[107,108]$. Kaise et al. used the triad ME-NBI findings, including fine mucosal surface disappearance, microvascular dilation, and heterogeneity, to diagnose EGC with sensitivity and specificity of $69.1 \%$ and $85.3 \%$, respectively, and the AUC of ROC in multivariate analysis was 0.86 [108]. Yao et al. described a simple classification system, called "VS classification", by regularity and presence of vascular and microsurface patterns under ME-NBI [109]. In this study, irregular vascularity and irregularity or absence of microsurface pattern with well-delineated DL were highly associated with carcinomatous gastric mucosa [109]. Sometimes, white opaque substance (WOS) within epithelium obscures observation of microvascular pattern under MENBI. WOS can be seen more frequently in adenoma than in carcinoma (78\% versus 43\%) [110]. For gastric neoplasia of type 0 -IIa type with either WOS with a regular distribution or a regular microvascular pattern, the sensitivity and specificity for discriminating adenoma from carcinoma were $94 \%$ and $96 \%$, respectively [110].

4.2. Flexible Spectral Imaging Color Enhancement. Mouri et al. has analyzed the wavelengths to generate the maximum difference of the spectral reflectance between the normal gastric mucosa and the EGC, and the result has shown that setting the wavelength at $530 \mathrm{~nm}$ of FICE observation resulted in an improvement in the visualization of the EGCs [111]. The diagnostic accuracy of extent of gastric cancer using FICE is superior to that using conventional WLI system [112], and with selection of specific reflectance spectrum, FICE is useful in discriminating among nonneoplastic lesions, adenoma, and EGC [28].

4.3. Autofluorescence Imaging System. An earlier study showed that AFI had high sensitivity (96.4\%) but low specificity $(49.1 \%)$ to diagnose gastric neoplasm [113]. Uedo and his coworkers used a case series study to conclude that AFI had lower diagnostic accuracy (68\%) than that of chromoendoscopy (91\%), but with an advantage over standard WLI (36\%) in diagnosis of EGC [29]. Moreover, open-type, 
atrophic fundic gastritis diagnosed by AFI was significantly associated with the development of metachronous gastric cancer (hazard ratio 4.88, 95\% CI 1.32-18.2) after Hp eradication therapy [114]. However, there are some limitations for AFI to diagnose EGC accurately. Ulcerations, inflammation, or scars may cause overdiagnosis in the AF observation [29], and AFI tends to overestimate the size of gastric neoplasm [115].

\subsection{Confocal Laser Endomicroscopy and Endocytoscopy}

4.4.1. Hp-Associated Gastritis. CLE with topical acriflavine was firstly used to identify $H p$ infection in a patient in 2005, and the bacterium which uptakes acriflavine ex vivo was seen as bright dots [116]. A prospective study uses three features under acriflavine-guided CLE, including white spots, neutrophils, and microabscesses, to detect $H p$ infection with the accuracy, sensitivity and specificity, of $92.8 \%, 89.2 \%$, and $95.7 \%$, respectively [117]. According to the established CLE criteria added with the presence of fluorescein leakage, histological severity of $\mathrm{Hp}$-associated gastritis was graded [118]. The sensitivity and specificity of CLE were $82.9 \%$ and $90.9 \%$ for the diagnosis of $\mathrm{Hp}$ infection, $94.6 \%$ and 97.4\% for predicting gastric normal mucosa, $98.5 \%$ and 94.6\% for predicting active inflammation, $92.9 \%$ and $95.2 \%$ for predicting atrophy of glands, and $98.6 \%$ and $100 \%$ for diagnosing IM, respectively [118].

4.4.2. Gastric Intestinal Metaplasia. A prospective study used the histopathological criteria to diagnose GIM by CLE. These criteria included goblet cells, columnar absorptive cells and brush border, and villiform foveolar epithelium [119]. CLE can differentiate complete metaplasia (sensitivity/specificity 68.03/89.66\%) from incomplete metaplasia (sensitivity/specificity $68.42 / 83.41 \%$ ), with the latter being closely associated with gastric cancer, according to the shape of the goblet cells, the presence of absorptive cells or brush border, and the architecture of vessels and crypts [119]. In another prospective study conducted by the same group, CLE found out 189 patients with gastric IM in 1572 "endoscopic normal-looking mucosa” patients by WLI system [120]. Realtime iCLE diagnosis had a higher sensitivity (88.9\%), specificity $(99.3 \%)$, and accuracy (98.8\%) for gastric superficial cancer/HGIN lesions than WLI diagnosis (sensitivity, 72.2\%; specificity, 95.1\%; accuracy, 94.1\%) $(P<0.05)[120]$.

4.4.3. Early Gastric Cancer. Kakeji et al. reported ex vivo tissues of 27 gastric cancers using CLE had sensitivity, specificity, and accuracy of $88-92.6 \%, 100 \%$, and $94.4-$ $96.3 \%$ (by endoscopists and pathologist), respectively [121]. Kitabatake et al. used in vivo CLE images to interpret by pathologists, and the results were compared with histology. The diagnostic accuracy was 94.2-96.2\% [122]. A prospective comparative study has demonstrated higher accuracy of CLE diagnosis of gastric adenomas and adenocarcinomas than that of endoscopic biopsy (94.2\% versus $85.7 \%, P=0.031$ ) [123]. The use of CLE optical biopsy could potentially replace conventional histological biopsy.
In field of EC for stomach, only a case report or a small sample size study was reported $[124,125]$. Eberl et al. showed that the sensitivity and specificity of EC were lower in gastric lesions (56\% and 89\%) compared to those in esophagus and colon (about 79-80\%, and 90-100\%) [124]. The poor diagnostic performance of EC may come from gastric mucus secretion [124]. Good quality of high magnified images by CLE or EC cannot always be obtained and there is still a long way to go for clinical application in mass screening of GI tract neoplasia.

4.5. Chromoendoscopy. Indigo carmine, a nonabsorbable dye, had been used in imaging of gastric lesions for more than 30 years, and it is still a useful technique to detect gastric neoplasia and determine the demarcation line before endoscopic resection $[126,127]$. Acetic acid was also useful in distinguishing dysplastic from nondysplastic lesions based on the difference in duration of acetowhitening reaction between neoplastic and nonneoplastic mucosa. The whitening reaction disappeared less than 5 seconds in invasive carcinoma compared to that about 90 seconds in nonneoplastic mucosa and low-grade adenoma [128]. Tanaka et al. further categorized gastric lesions into five types according to enhancedmagnification endoscopy findings following $1.5 \%$ acetic acid instillation, and all of the signet-ring cell carcinomas and poorly differentiated tubular adenocarcinomas have shown irregular arrangement or destructive surface pattern [129]. When combining chromoendoscopy with zoom endoscopy, the microsurface and microvascular structures could be visualized more easily. Magnification chromoendoscopy with $1 \%$ methylene blue was validated to assess premalignant gastric lesions (chronic atrophic gastritis with or without intestinal metaplasia) with sensitivity and specificity of $100 \%$ and 99\%, respectively [130]. Dinis-Ribeiro et al. also used magnification chromoendoscopy with methylene blue to classify pit pattern of gastric lesions into 10 subgroups [131]. The classification system had the specificity and NPV of $81 \%$ and $99 \%$, respectively, to diagnose dysplasia. Congo red alone, or combined with methylene blue, has also been applied in chromoendoscopy $[132,133]$. The correct diagnosis of synchronous multiple loci of EGC by Congo redmethylene blue chromoendoscopy could be higher than that of conventional WLI endoscopy (28.3\% versus $88.9 \%$ ) [133]. Prolonged procedure time by staining method is still the common problem for chromoendoscopy screening of GI neoplasia.

\section{Conclusions}

The early detection of precancerous or early cancerous lesions of gastrointestinal tract is of utmost importance to timely curative treatment, improved overall outcome, and the maintenance of a good quality of life. Therefore, visualizeing the invisibles by endoscopists based on the utilization of IEEs on cancer screening could solve problems from missing lesions by conventional WLI endoscopy. Optical imaging technology has high diagnostic accuracy, and image reconstruction technique could provide similar imaging with histopathological findings. After obtaining universal standardized imaging 
method at high magnification level and images with good quality, optical biopsy could replace histological biopsy in the near future.

\section{Acknowledgments}

No funding in support of the work is to be declared, and the authors report no conflict of interests. The authors thank Dr. Wei-Fan Hsu from the Far Eastern Memorial Hospital, New Taipei City, Taiwan, and Dr. Chih-Hsien Wang from the National Taiwan University Hospital, Yun-Lin Branch, Taiwan, for assistance in collection of data.

\section{References}

[1] P. L. B. Bolye, World Cancer Report, IARC Press, Lyon, France, 2008.

[2] A. Jemal, F. Bray, M. M. Center, J. Ferlay, E. Ward, and D. Forman, "Global cancer statistics," CA: Cancer Journal for Clinicians, vol. 61, no. 2, pp. 69-90, 2011.

[3] K. K. Wang and R. E. Sampliner, "Updated guidelines 2008 for the diagnosis, surveillance and therapy of Barrett's esophagus," American Journal of Gastroenterology, vol. 103, no. 3, pp. 788797, 2008.

[4] J. Theisen, H. J. Stein, H. J. Dittler et al., "Preoperative chemotherapy unmasks underlying Barrett's mucosa in patients with adenocarcinoma of the distal esophagus," Surgical Endoscopy and Other Interventional Techniques, vol. 16, no. 4, pp. 671-673, 2002.

[5] F. Hvid-Jensen, L. Pedersen, A. M. Drewes, H. T. Sørensen, and P. Funch-Jensen, "Incidence of adenocarcinoma among patients with Barrett's esophagus," The New England Journal of Medicine, vol. 365, no. 15, pp. 1375-1383, 2011.

[6] J. L. Whiting, A. Sigurdsson, D. C. Rowlands, M. T. Hallissey, and J. W. L. Fielding, "The long term results of endoscopic surveillance of premalignant gastric lesions," Gut, vol. 50, no. 3, pp. 378-381, 2002.

[7] S. M. Everett and A. T. R. Axon, "Early gastric cancer in Europe," Gut, vol. 41, no. 2, pp. 142-150, 1997.

[8] M. Kodama and T. Kakegawa, "Treatment of superficial cancer of the esophagus: a summary of responses to a questionnaire on superficial cancer of the esophagus in Japan," Surgery, vol. 123, no. 4, pp. 432-439, 1998.

[9] K. Takubo, J. Aida, M. Sawabe et al., "Early squamous cell carcinoma of the oesophagus: the Japanese viewpoint," Histopathology, vol. 51, no. 6, pp. 733-742, 2007.

[10] T. Gotoda, A. Yanagisawa, M. Sasako et al., "Incidence of lymph node metastasis from early gastric cancer: estimation with a large number of cases at two large centers," Gastric Cancer, vol. 3, no. 4, pp. 219-225, 2000.

[11] T. Hirasawa, T. Gotoda, S. Miyata et al., "Incidence of lymph node metastasis and the feasibility of endoscopic resection for undifferentiated-type early gastric cancer," Gastric Cancer, vol. 12, no. 3, pp. 148-152, 2009.

[12] M. Tada, M. Shimada, and F. Murakami, "Development of the strip-off biopsy," Gastroenterological Endoscopy, vol. 26, no. 6, pp. 833-839, 1984.

[13] T. Gotoda, "Endoscopic resection of early gastric cancer," Gastric Cancer, vol. 10, no. 1, pp. 1-11, 2007.

[14] H. Inoue, K. Takeshita, H. Hori, Y. Muraoka, H. Yoneshima, and M. Endo, "Endoscopic mucosal resection with a cap-fitted panendoscope for esophagus, stomach, and colon mucosal lesions," Gastrointestinal Endoscopy, vol. 39, no. 1, pp. 58-62, 1993.

[15] M. Akiyama, M. Ota, H. Nakajima, K. Yamagata, and A. Munakata, "Endoscopic mucosal resection of gastric neoplasms using a ligating device," Gastrointestinal Endoscopy, vol. 45, no. 2, pp. 182-186, 1997.

[16] T. Oyama, A. Tomori, K. Hotta et al., "Endoscopic submucosal dissection of early esophageal cancer," Clinical Gastroenterology and Hepatology, vol. 3, no. 7, pp. S67-S70, 2005.

[17] M. Kato, "Endoscopic submucosal dissection (ESD) is being accepted as a new procedure of endoscopic treatment of early gastric cancer," Internal Medicine, vol. 44, no. 2, pp. 85-86, 2005.

[18] J. Lian, S. Chen, Y. Zhang, and F. Qiu, "A meta-analysis of endoscopic submucosal dissection and EMR for early gastric cancer," Gastrointestinal Endoscopy, vol. 76, no. 4, pp. 763-770, 2012.

[19] T. Kaltenbach, Y. Sano, S. Friedland, and R. Soetikno, "American Gastroenterological Association (AGA) Institute technology assessment on image-enhanced endoscopy," Gastroenterology, vol. 134, no. 1, pp. 327-340, 2008.

[20] K. Gono, T. Obi, M. Yamaguchi et al., "Appearance of enhanced tissue features in narrow-band endoscopic imaging," Journal of Biomedical Optics, vol. 9, no. 3, pp. 568-577, 2004.

[21] S. Okuyama, M. Ohata, H. Orimo, T. Fujita, and M. Yoshikawa, "FGS-ML," Gastrointestinal Endoscopy, vol. 9, pp. 42-43, 1967.

[22] M. Muto, T. Horimatsu, Y. Ezoe, S. Morita, and S. Miyamoto, "Improving visualization techniques by narrow band imaging and magnification endoscopy," Journal of Gastroenterology and Hepatology, vol. 24, no. 8, pp. 1333-1346, 2009.

[23] D. B. Nelson, K. P. Block, J. J. Bosco et al., "High resolution and high-magnification endoscopy," Gastrointestinal Endoscopy, vol. 52, no. 6, pp. 864-866, 2000.

[24] T. Yoshida, H. Inoue, S. Usui, H. Satodate, N. Fukami, and S. E. Kudo, "Narrow-band imaging system with magnifying endoscopy for superficial esophageal lesions," Gastrointestinal Endoscopy, vol. 59, no. 2, pp. 288-295, 2004.

[25] K. Kubo and M. A. Fujino, "Ultra-high magnification endoscopy of the normal esophageal mucosa," Gastrointestinal Endoscopy, vol. 46, no. 1, pp. 96-97, 1997.

[26] J. Pohl, A. May, T. Rabenstein, O. Pech, and C. Ell, “Computed virtual chromoendoscopy: a new tool for enhancing tissue surface structures," Endoscopy, vol. 39, no. 1, pp. 80-83, 2007.

[27] M. Inoue, Y. Miyake, T. Odaka et al., "Objective evaluation of visibility in virtual chromoendoscopy for esophageal squamous carcinoma using a color difference formula," Journal of Biomedical Optics, vol. 15, no. 5, Article ID 056019, 2010.

[28] S. W. Jung, K. S. Lim, J. U. Lim et al., "Flexible spectral imaging color enhancement (FICE) is useful to discriminate among non-neoplastic lesion, adenoma, and cancer of stomach," Digestive Diseases and Sciences, vol. 56, no. 10, pp. 2879-2886, 2011.

[29] N. Uedo, H. Iishi, M. Tatsuta et al., "A novel videoendoscopy system by using autofluorescence and reflectance imaging for diagnosis of esophagogastric cancers," Gastrointestinal Endoscopy, vol. 62, no. 4, pp. 521-528, 2005.

[30] J. Haringsma, G. N. J. Tytgat, H. Yano et al., "Autofluorescence endoscopy: feasibility of detection of GI neoplasms unapparent to white light endoscopy with an evolving technology," Gastrointestinal Endoscopy, vol. 53, no. 6, pp. 642-650, 2001.

[31] H. Liu, Y. Q. Li, T. Yu et al., "Confocal endomicroscopy for in vivo detection of microvascular architecture in normal and 
malignant lesions of upper gastrointestinal tract," Journal of Gastroenterology and Hepatology, vol. 23, no. 1, pp. 56-61, 2008.

[32] M. B. Wallace and P. Fockens, "Probe-based confocal laser endomicroscopy," Gastroenterology, vol. 136, no. 5, pp. 15091513, 2009.

[33] R. S. Kwon, L. M. Wong Kee Song, D. G. Adler et al., "Endocytoscopy," Gastrointestinal Endoscopy, vol. 70, no. 4, pp. 610-613, 2009.

[34] H. Inoue, S. E. Kudo, and A. Shiokawa, "Technology insight: laser-scanning confocal microscopy and endocytoscopy for cellular observation of the gastrointestinal tract," Nature Clinical Practice Gastroenterology and Hepatology, vol. 2, no. 1, pp. 31-37, 2005.

[35] H. Neumann, F. S. Fuchs, M. Vieth et al., "Review article: in vivo imaging by endocytoscopy," Alimentary Pharmacology and Therapeutics, vol. 33, no. 11, pp. 1183-1193, 2011.

[36] M. V. Sivak, K. Kobayashi, J. A. Izatt et al., "High-resolution endoscopic imaging of the GI tract using optical coherence tomography," Gastrointestinal Endoscopy, vol. 51, no. 4, pp. 474479, 2000.

[37] G. Zuccaro, N. Gladkova, J. Vargo et al., "Optical coherence tomography of the esophagus and proximal stomach in health and disease," American Journal of Gastroenterology, vol. 96, no. 9, pp. 2633-2639, 2001.

[38] W. Y. Cho, J. Y. Jang, and D. H. Lee, "Recent advances in imageenhanced endoscopy," Clinical Endoscopy, vol. 44, no. 2, pp. 6575, 2011.

[39] S. Kodashima and M. Fujishiro, "Novel image-enhanced endoscopy with i-scan technology," World Journal of Gastroenterology, vol. 16, no. 9, pp. 1043-1049, 2010.

[40] M. Kida, K. Kobayashi, and K. Saigenji, "Routine chromoendoscopy for gastrointestinal diseases: indications revised," Endoscopy, vol. 35, no. 7, pp. 590-596, 2003.

[41] S. Toriie, Y. Akasaka, and K. Yamaguchi, "New trial for endoscopical observation of esophagus by dye spraying method," GEN, vol. 30, no. 3-4, pp. 159-165, 1976.

[42] Y. Shimizu, T. Omori, A. Yokoyama et al., "Endoscopic diagnosis of early squamous neoplasia of the esophagus with iodine staining: high-grade intra-epithelial neoplasia turns pink within a few minutes," Journal of Gastroenterology and Hepatology, vol. 23, no. 4, pp. 546-550, 2008.

[43] H. Kondo, H. Fukuda, H. Ono et al., "Sodium thiosulfate solution spray for relief of irritation caused by Lugol's stain in chromoendoscopy," Gastrointestinal Endoscopy, vol. 53, no. 2, pp. 199-202, 2001.

[44] G. Longcroft-Wheaton, M. Duku, R. Mead, D. Poller, and P. Bhandari, "Acetic acid spray is an effective tool for the endoscopic detection of neoplasia in patients with barrett's esophagus," Clinical Gastroenterology and Hepatology, vol. 8, no. 10, pp. 843-847, 2010.

[45] J. R. Olliver, C. P. Wild, P. Sahay, S. Dexter, and L. J. Hardie, "Chromoendoscopy with methylene blue and associated DNA damage in Barrett's oesophagus," The Lancet, vol. 362, no. 9381, pp. 373-374, 2003.

[46] M. Dinis-Ribeiro and L. Moreira-Dias, "There is no clinical evidence of consequences after methylene blue chromoendoscopy," Gastrointestinal Endoscopy, vol. 67, no. 7, p. 1209, 2008.

[47] C. S. Chung, Y. C. Lee, C. P. Wang et al., "Secondary prevention of esophageal squamous cell carcinoma in areas where smoking, alcohol, and betel quid chewing are prevalent," Journal of the Formosan Medical Association, vol. 109, no. 6, pp. 408-421, 2010.
[48] C. T. Lee, C. Y. Chang, Y. C. Lee et al., "Narrow-band imaging with magnifying endoscopy for the screening of esophageal cancer in patients with primary head and neck cancers," Endoscopy, vol. 42, no. 8, pp. 613-619, 2010.

[49] Y. C. Lee, C. P. Wang, C. C. Chen et al., "Transnasal endoscopy with narrow-band imaging and Lugol staining to screen patients with head and neck cancer whose condition limits oral intubation with standard endoscope (with video)," Gastrointestinal Endoscopy, vol. 69, no. 3, pp. 408-417, 2009.

[50] H. Inoue, T. Honda, K. Nagai et al., "Ultra-high magnification endoscopic observation of carcinoma in situ of the esophagus," Digestive Endoscopy, vol. 9, no. 1, pp. 16-18, 1997.

[51] N. Uedo, M. Fujishiro, K. Goda et al., "Role of narrow band imaging for diagnosis of early-stage esophagogastric cancer: current consensus of experienced endoscopists in Asia-Pacific region," Digestive Endoscopy, vol. 23, no. 1, pp. 58-71, 2011.

[52] R. Ishihara, T. Inoue, N. Uedo et al., "Significance of each narrow-band imaging finding in diagnosing squamous mucosal high-grade neoplasia of the esophagus," Journal of Gastroenterology and Hepatology, vol. 25, no. 8, pp. 1410-1415, 2010.

[53] H. Inoue, "Endoscopic diagnosis of tissue atypism (EA) in the pharyngeal and esophageal squamous epithelium; IPCL pattern classification and ECA classification," Kyobu Geka, vol. 60, no. 8, pp. 768-775, 2007.

[54] Y. Kumagai, H. Inoue, K. Nagai, T. Kawano, and T. Iwai, "Magnifying endoscopy, stereoscopic microscopy, and the microvascular architecture of superficial esophageal carcinoma," Endoscopy, vol. 34, no. 5, pp. 369-375, 2002.

[55] M. Arima, M. Tada, and H. Arima, "Evaluation of microvascular patterns of superficial esophageal cancers by magnifying endoscopy," Esophagus, vol. 2, no. 4, pp. 191-197, 2005.

[56] G. K. Anagnostopoulos, K. Yao, P. Kaye, C. J. Hawkey, and K. Ragunath, "Novel endoscopic observation in Barrett's oesophagus using high resolution magnification endoscopy and narrow band imaging," Alimentary Pharmacology and Therapeutics, vol. 26, no. 3, pp. 501-507, 2007.

[57] R. Singh, G. K. Anagnostopoulos, K. Yao et al., "Narrow-band imaging with magnification in Barrett's esophagus: validation of a simplified grading system of mucosal morphology patterns against histology," Endoscopy, vol. 40, no. 6, pp. 457-463, 2008.

[58] J. Mannath, V. Subramanian, C. J. Hawkey, and K. Ragunath, "Narrow band imaging for characterization of high grade dysplasia and specialized intestinal metaplasia in Barretts esophagus: a meta-analysis," Endoscopy, vol. 42, no. 5, pp. 351$359,2010$.

[59] A. Oka, Y. Amano, R. Kusunoki et al., "Superficial esophageal cancer observed with the PillCam ESO 2 in combination with the flexible spectral imaging color enhancement system," Digestive Endoscopy, vol. 23, no. 2, pp. 195-196, 2011.

[60] H. Osawa, H. Yamamoto, N. Yamada et al., "Diagnosis of endoscopic Barrett's esophagus by transnasal flexible spectral imaging color enhancement," Journal of Gastroenterology, vol. 44, no. 11, pp. 1125-1132, 2009.

[61] J. Pohl, A. May, T. Rabenstein et al., "Comparison of computed virtual chromoendoscopy and conventional chromoendoscopy with acetic acid for detection of neoplasia in Barrett's esophagus," Endoscopy, vol. 39, no. 7, pp. 594-598, 2007.

[62] Y. Yoshida, K. Goda, H. Tajiri, M. Urashima, N. Yoshimura, and T. Kato, "Assessment of novel endoscopic techniques for visualizing superficial esophageal squamous cell carcinoma: autofluorescence and narrow-band imaging," Diseases of the Esophagus, vol. 22, no. 5, pp. 439-446, 2009. 
[63] R. Ishihara, T. Inoue, N. Hanaoka et al., "Autofluorescence imaging endoscopy for screening of esophageal squamous mucosal high-grade neoplasia: a phase II study," Journal of Gastroenterology and Hepatology, vol. 27, no. 1, pp. 86-90, 2012.

[64] M. Panjehpour, B. F. Overholt, T. Vo-Dinh, R. C. Haggitt, D. H. Edwards, and F. P. I. Buckley, "Endoscopic fluorescence detection of high-grade dysplasia in Barrett's esophagus," Gastroenterology, vol. 111, no. 1, pp. 93-101, 1996.

[65] S. Brand, T. D. Wang, K. T. Schomacker et al., "Detection of high-grade dysplasia in Barrett's esophagus by spectroscopy measurement of 5-aminolevulinic acid-induced protoporphyrin IX fluorescence," Gastrointestinal Endoscopy, vol. 56, no. 4, pp. 479-487, 2002.

[66] M. A. Kara, F. P. Peters, P. Fockens, F. J. W. ten Kate, and J. J. G. H. M. Bergman, "Endoscopic video-autofluorescence imaging followed by narrow band imaging for detecting early neoplasia in Barrett's esophagus," Gastrointestinal Endoscopy, vol. 64, no. 2, pp. 176-185, 2006.

[67] W. L. Curvers, L. A. Herrero, M. B. Wallace et al., "Endoscopic tri-modal imaging is more effective than standard endoscopy in identifying early-stage neoplasia in Barrett's esophagus," Gastroenterology, vol. 139, no. 4, pp. 1106-el, 2010.

[68] W. L. Curvers, R. Singh, L. M. W. K. Song et al., "Endoscopic tri-modal imaging for detection of early neoplasia in Barrett's oesophagus: a multi-centre feasibility study using highresolution endoscopy, autofluorescence imaging and narrow band imaging incorporated in one endoscopy system," Gut, vol. 57, no. 2, pp. 167-172, 2008.

[69] W. L. Curvers, F. G. Van Vilsteren, L. C. Baak et al., "Endoscopic trimodal imaging versus standard video endoscopy for detection of early Barrett's neoplasia: a multicenter, randomized, crossover study in general practice," Gastrointestinal Endoscopy, vol. 73, no. 2, pp. 195-203, 2011.

[70] H. Liu, Y. Q. Li, T. Yu et al., "Confocal laser endomicroscopy for superficial esophageal squamous cell carcinoma," Endoscopy, vol. 41, no. 2, pp. 99-106, 2009.

[71] O. Pech, T. Rabenstein, H. Manner et al., "Confocal laser endomicroscopy for in vivo diagnosis of early squamous cell carcinoma in the esophagus," Clinical Gastroenterology and Hepatology, vol. 6, no. 1, pp. 89-94, 2008.

[72] H. Inoue, K. Sasajima, M. Kaga et al., "Endoscopic in vivo evaluation of tissue atypia in the esophagus using a newly designed intergrated endocytoscope: a pilot trial," Endoscopy, vol. 38, no. 9, pp. 891-895, 2006.

[73] Y. Kumagai, K. Kawada, S. Yamazaki et al., "Endocytoscopic observation for esophageal squamous cell carcinoma: can biopsy histology be omitted?" Diseases of the Esophagus, vol. 22, no. 6, pp. 505-512, 2009.

[74] M. Fujishiro, K. Takubo, Y. Sato et al., "Potential and present limitation of endocytoscopy in the diagnosis of esophageal squamous-cell carcinoma: a multicenter ex vivo pilot study," Gastrointestinal Endoscopy, vol. 66, no. 3, pp. 551-555, 2007.

[75] R. Kiesslich, L. Gossner, M. Goetz et al., "In vivo histology of Barrett's esophagus and associated neoplasia by confocal laser endomicroscopy," Clinical Gastroenterology and Hepatology, vol. 4, no. 8, pp. 979-987, 2006.

[76] K. B. Dunbar, P. Okolo, E. Montgomery, and M. I. Canto, "Confocal laser endomicroscopy in Barrett's esophagus and endoscopically inapparent Barrett's neoplasia: a prospective, randomized, double-blind, controlled, crossover trial," Gastrointestinal Endoscopy, vol. 70, no. 4, pp. 645-654, 2009.
[77] P. Sharma, A. R. Meining, E. Coron et al., "Real-time increased detection of neoplastic tissue in Barrett's esophagus with probebased confocal laser endomicroscopy: final results of an international multicenter, prospective, randomized, controlled trial," Gastrointestinal Endoscopy, vol. 74, no. 3, pp. 465-472, 2011.

[78] M. Wallace, G. Y. Lauwers, Y. Chen et al., "Miami classification for probe-based confocal laser endomicroscopy," Endoscopy, vol. 43, no. 10, pp. 882-891, 2011.

[79] M. Bajbouj, M. Vieth, T. Rösch et al., "Probe-based confocal laser endomicroscopy compared with standard four-quadrant biopsy for evaluation of neoplasia in Barretts esophagus," Endoscopy, vol. 42, no. 6, pp. 435-440, 2010.

[80] H. Bertani, M. Frazzoni, E. Dabizzi et al., "Improved detection of incident dysplasia by probe-based confocal laser endomicroscopy in a Barrett's esophagus surveillance program," Digestive Diseases and Sciences, vol. 58, no. 1, pp. 188-193, 2012.

[81] H. Pohl, M. Koch, A. Khalifa et al., "Evaluation of endocytoscopy in the surveillance of patients with Barrett's esophagus," Endoscopy, vol. 39, no. 6, pp. 492-496, 2007.

[82] W. Hatta, K. Uno, T. Koike et al., "Optical coherence tomography for the staging of tumor infiltration in superficial esophageal squamous cell carcinoma," Gastrointestinal Endoscopy, vol. 71, no. 6, pp. 899-906, 2010.

[83] W. Hatta, K. Uno, T. Koike et al., "A prospective comparative study of optical coherence tomography and EUS for tumor staging of superficial esophageal squamous cell carcinoma," Gastrointestinal Endoscopy, vol. 76, no. 3, pp. 548-555, 2012.

[84] J. M. Poneros, S. Brand, B. E. Bouma, G. J. Tearney, C. C. Compton, and N. S. Nishioka, "Diagnosis of specialized intestinal metaplasia by optical coherence tomography," Gastroenterology, vol. 120, no. 1, pp. 7-12, 2001.

[85] G. Isenberg, M. V. Sivak, A. Chak et al., "Accuracy of endoscopic optical coherence tomography in the detection of dysplasia in Barrett's esophagus: a prospective, double-blinded study," Gastrointestinal Endoscopy, vol. 62, no. 6, pp. 825-831, 2005.

[86] X. Qi, Y. Pan, M. V. Sivak, J. E. Willis, G. Isenberg, and A. M. Rollins, "Image analysis for classification of dysplasia in Barrett's esophagus using endoscopic optical coherence tomography," Biomedical Optics Express, vol. 1, no. 3, pp. 825-847, 2010.

[87] T.-H. Tsai, C. Zhou, H.-C. Lee et al., "Comparison of tissue architectural changes between radiofrequency ablation and cryospray ablation in Barrett's esophagus using endoscopic three-dimensional optical coherence tomography," Gastroenterology Research and Practice, vol. 2012, Article ID 684832, 8 pages, 2012.

[88] A. Hoffman, N. Basting, M. Goetz et al., "High-definition endoscopy with i-Scan and Lugol's solution for more precise detection of mucosal breaks in patients with reflux symptoms," Endoscopy, vol. 41, no. 2, pp. 107-112, 2009.

[89] M. S. Kim, S. R. Choi, M. H. Roh et al., "Efficacy of I-scan endoscopy in the diagnosis of gastroesophageal reflux disease with minimal change," Clinical Endoscopy, vol. 44, no. 1, pp. 2732, 2011.

[90] S. M. Dawsey, D. E. Fleischer, G. Q. Wang et al., "Mucosal iodine staining improves endoscopic visualization of squamous dysplasia and squamous cell carcinoma of the esophagus in Linxian, China," Cancer, vol. 83, no. 2, pp. 220-231, 1998.

[91] M. I. F. Canto, S. Setrakian, R. E. Petras, E. Blades, A. Chak, and M. Sivak, "Methylene blue selectively stains intestinal metaplasia in Barrett's esophagus," Gastrointestinal Endoscopy, vol. 44, no. 1, pp. 1-7, 1996. 
[92] R. Kiesslich, M. Hahn, G. Herrmann, and M. Jung, "Screening for specialized columnar epithelium with methylene blue: chromoendoscopy in patients with Barrett's esophagus and a normal control group," Gastrointestinal Endoscopy, vol. 53, no. 1, pp. 47-52, 2001.

[93] T. Endo, T. Awakawa, H. Takahashi et al., "Classification of Barrett's epithlium by magnifying endoscopy," Gastrointestinal Endoscopy, vol. 55, no. 6, pp. 641-647, 2002.

[94] K. Ragunath, N. Krasner, V. S. Raman, M. T. Haqqani, and W. Y. Cheung, "A randomized, prospective cross-over trial comparing methylene blue-directed biopsy and conventional random biopsy for detecting intestinal metaplasia and dysplasia in Barrett's esophagus," Endoscopy, vol. 35, no. 12, pp. 998-1003, 2003.

[95] S. Ngamruengphong, V. K. Sharma, and A. Das, "Diagnostic yield of methylene blue chromoendoscopy for detecting specialized intestinal metaplasia and dysplasia in Barrett's esophagus: a meta-analysis," Gastrointestinal Endoscopy, vol. 69, no. 6, pp. 1021-1028, 2009.

[96] A. Hoffman, R. Kiesslich, A. Bender et al., "Acetic acid-guided biopsies after magnifying endoscopy compared with random biopsies in the detection of Barrett's esophagus: a prospective randomized trial with crossover design," Gastrointestinal Endoscopy, vol. 64, no. 1, pp. 1-8, 2006.

[97] P. J. Fortun, G. K. Anagnostopoulos, P. Kaye et al., "Acetic acidenhanced magnification endoscopy in the diagnosis of specialized intestinal metaplasia, dysplasia and early cancer in Barrett's oesophagus," Alimentary Pharmacology and Therapeutics, vol. 23, no. 6, pp. 735-742, 2006.

[98] J. L. Vázquez-Iglesias, P. Alonso-Aguirre, M. T. Diz-Lois, M. A. Vázquez-Millán, A. Álvarez, and M. J. Lorenzo, "Acetic acid allows effective selection of areas for obtaining biopsy samples in Barrett's esophagus," European Journal of Gastroenterology and Hepatology, vol. 19, no. 3, pp. 187-193, 2007.

[99] A. A. Alaboudy, A. Elbahrawy, S. Matsumoto, and A. Yoshizawa, "Conventional narrow-band imaging has good correlation with histopathological severity of Helicobacter pylori gastritis," Digestive Diseases and Sciences, vol. 56, no. 4, pp. 1127-1130, 2011.

[100] G. K. Anagnostopoulos, K. Yao, P. Kaye et al., "High-resolution magnification endoscopy can reliably identify normal gastric mucosa, Helicobacter pylori-associated gastritis, and gastric atrophy," Endoscopy, vol. 39, no. 3, pp. 202-207, 2007.

[101] T. Tahara, T. Shibata, M. Nakamura et al., "Gastric mucosal pattern by using magnifying narrow-band imaging endoscopy clearly distinguishes histological and serological severity of chronic gastritis," Gastrointestinal Endoscopy, vol. 70, no. 2, pp. 246-253, 2009.

[102] K. Yagi, A. Nakamura, and A. Sekine, "Comparison between magnifying endoscopy and histological, culture and urease test findings from the gastric mucosa of the corpus," Endoscopy, vol. 34, no. 5, pp. 376-381, 2002.

[103] M. M. Walker, "Is intestinal metaplasia of the stomach reversible?" Gut, vol. 52, no. 1, pp. 1-4, 2003.

[104] N. Uedo, R. Ishihara, H. Iishi et al., "A new method of diagnosing gastric intestinal metaplasia: narrow-band imaging with magnifying endoscopy," Endoscopy, vol. 38, no. 8, pp. 819824, 2006.

[105] P. Pimentel-Nunes, M. Dinis-Ribeiro, J. B. Soares et al., "A multicenter validation of an endoscopic classification with narrow band imaging for gastric precancerous and cancerous lesions," Endoscopy, vol. 44, no. 3, pp. 236-246, 2012.
[106] Y. Otsuka, Y. Niwa, N. Ohmiya et al., "Usefulness of magnifying endoscopy in the diagnosis of early gastric cancer," Endoscopy, vol. 36, no. 2, pp. 165-169, 2004.

[107] T. Nakayoshi, H. Tajiri, K. Matsuda, M. Kaise, M. Ikegami, and H. Sasaki, "Magnifying endoscopy combined with narrow band imaging system for early gastric cancer: correlation of vascular pattern with histopathology," Endoscopy, vol. 36, no. 12, pp. 1080-1084, 2004.

[108] M. Kaise, M. Kato, M. Urashima et al., "Magnifying endoscopy combined with narrow-band imaging for differential diagnosis of superficial depressed gastric lesions," Endoscopy, vol. 41, no. 4, pp. 310-315, 2009.

[109] K. Yao, G. K. Anagnostopoulos, and K. Ragunath, "Magnifying endoscopy for diagnosing and delineating early gastric cancer," Endoscopy, vol. 41, no. 5, pp. 462-467, 2009.

[110] K. Yao, A. Iwashita, H. Tanabe et al., "White opaque substance within superficial elevated gastric neoplasia as visualized by magnification endoscopy with narrow-band imaging: a new optical sign for differentiating between adenoma and carcinoma," Gastrointestinal Endoscopy, vol. 68, no. 3, pp. 574-580, 2008.

[111] R. Mouri, S. Yoshida, S. Tanaka, S. Oka, M. Yoshihara, and K. Chayama, "Evaluation and validation of computed virtual chromoendoscopy in early gastric cancer," Gastrointestinal Endoscopy, vol. 69, no. 6, pp. 1052-1058, 2009.

[112] H. Osawa, H. Yamamoto, Y. Miura et al., "Diagnosis of extent of early gastric cancer using flexible spectral imaging color enhancement," World Journal of Gastrointestinal Endoscopy, vol. 16, no. 4, pp. 356-361, 2012.

[113] A. Ohkawa, H. Miwa, A. Namihisa et al., "Diagnostic performance of light-induced fluorescence endoscopy for gastric neoplasms," Endoscopy, vol. 36, no. 6, pp. 515-521, 2004.

[114] N. Hanaoka, N. Uedo, A. Shiotani et al., "Autofluorescence imaging for predicting development of metachronous gastric cancer after Helicobacter pylori eradication," Journal of Gastroenterology and Hepatology, vol. 25, no. 12, pp. 1844-1849, 2010.

[115] J. H. Lee, J. Y. Cho, M. G. Choi et al., "Usefulness of autofluorescence imaging for estimating the extent of gastric neoplastic lesions: a prospective multicenter study," Gut Liver, vol. 2, no. 3, pp. 174-179, 2008.

[116] R. Kiesslich, M. Goetz, J. Burg et al., "Diagnosing Helicobacter pylori in vivo by confocal laser endoscopy," Gastroenterology, vol. 128, no. 7, pp. 2119-2123, 2005.

[117] R. Ji, Y. Q. Li, X. M. Gu, T. Yu, X. L. Zuo, and C. J. Zhou, “Confocal laser endomicroscopy for diagnosis of Helicobacter pylori infection: a prospective study," Journal of Gastroenterology and Hepatology, vol. 25, no. 4, pp. 700-705, 2010.

[118] P. Wang, R. Ji, T. Yu et al., "Classification of histological severity of Helicobacter pylori - associated gastritis by confocal laser endomicroscopy," World Journal of Gastroenterology, vol. 16, no. 41, pp. 5203-5210, 2010.

[119] Y. T. Guo, Y. Q. Li, T. Yu et al., "Diagnosis of gastric intestinal metaplasia with confocal laser endomicroscopy in vivo: a prospective study," Endoscopy, vol. 40, no. 7, pp. 547-553, 2008.

[120] W. B. Li, X. L. Zuo, C. Q. Li et al., "Diagnostic value of confocal laser endomicroscopy for gastric superficial cancerous lesions," Gut, vol. 60, no. 3, pp. 299-306, 2011.

[121] Y. Kakeji, S. Yamaguchi, D. Yoshida et al., "Development and assessment of morphologic criteria for diagnosing gastric cancer using confocal endomicroscopy: an ex vivo and in vivo study," Endoscopy, vol. 38, no. 9, pp. 886-890, 2006. 
[122] S. Kitabatake, Y. Niwa, R. Miyahara et al., "Confocal endomicroscopy for the diagnosis of gastric cancer in vivo," Endoscopy, vol. 38, no. 11, pp. 1110-1114, 2006.

[123] S. R. Jeon, W. Y. Cho, S. Y. Jin, Y. K. Cheon, S. R. Choi, and J. Y. Cho, “. Optical biopsies by confocal endomicroscopy prevent additive endoscopic biopsies before endoscopic submucosal dissection in gastric epithelial neoplasias: a prospective, comparative study," Gastrointestinal Endoscopy, vol. 74, no. 4, pp. 772-780, 2011.

[124] T. Eberl, G. Jechart, A. Probst et al., "Can an endocytoscope system (ECS) predict histology in neoplastic lesions?" Endoscopy, vol. 39, no. 6, pp. 497-501, 2007.

[125] A. Fasoli, V. Pugliese, M. Furnari, B. Gatteschi, M. Truini, and E. Meroni, "Signet ring cell carcinoma of the stomach: correlation between endocytoscopy and histology," Endoscopy, vol. 41, pp. E65-66, 2009.

[126] T. Okabayashi, T. Gotoda, H. Kondo et al., "Usefulness of indigo carmine chromoendoscopy and endoscopic clipping for accurate preoperative assessment of proximal gastric cancer," Endoscopy, vol. 32, no. 10, p. S62, 2000.

[127] K. Ida, Y. Hashimoto, and S. Takeda, "Endoscopic diagnosis of gastric cancer with dye scattering," American Journal of Gastroenterology, vol. 63, no. 4, pp. 316-320, 1975.

[128] K. Yagi, Y. Aruga, A. Nakamura, A. Sekine, and H. Umezu, "The study of dynamic chemical magnifying endoscopy in gastric neoplasia CME," Gastrointestinal Endoscopy, vol. 62, no. 6, pp. 963-969, 2005.

[129] K. Tanaka, H. Toyoda, S. Kadowaki et al., "Features of early gastric cancer and gastric adenoma by enhanced-magnification endoscopy," Journal of Gastroenterology, vol. 41, no. 4, pp. 332338, 2006.

[130] M. Areia, P. Amaro, M. Dinis-Ribeiro et al., "External validation of a classification for methylene blue magnification chromoendoscopy in premalignant gastric lesions," Gastrointestinal Endoscopy, vol. 67, no. 7, pp. 1011-1018, 2008.

[131] M. Dinis-Ribeiro, A. da Costa-Pereira, C. Lopes et al., "Magnification chromoendoscopy for the diagnosis of gastric intestinal metaplasia and dysplasia," Gastrointestinal Endoscopy, vol. 57, no. 4, pp. 498-504, 2003.

[132] E. Toth, K. Sjolund, F. T. Fork, and C. Lindstrom, "Chronic atrophic fundic gastritis diagnosed by a modified Congo red test," Endoscopy, vol. 27, no. 9, pp. 654-658, 1995.

[133] H. Iishi, M. Tatsuta, and S. Okuda, "Diagnosis of simultaneous multiple gastric cancers by the endoscopic Congo redmethylene blue test," Endoscopy, vol. 20, no. 2, pp. 78-82, 1988. 


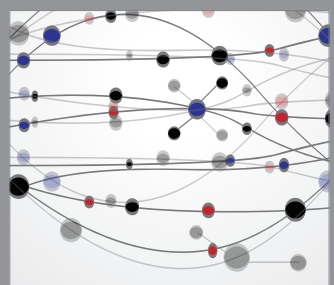

The Scientific World Journal
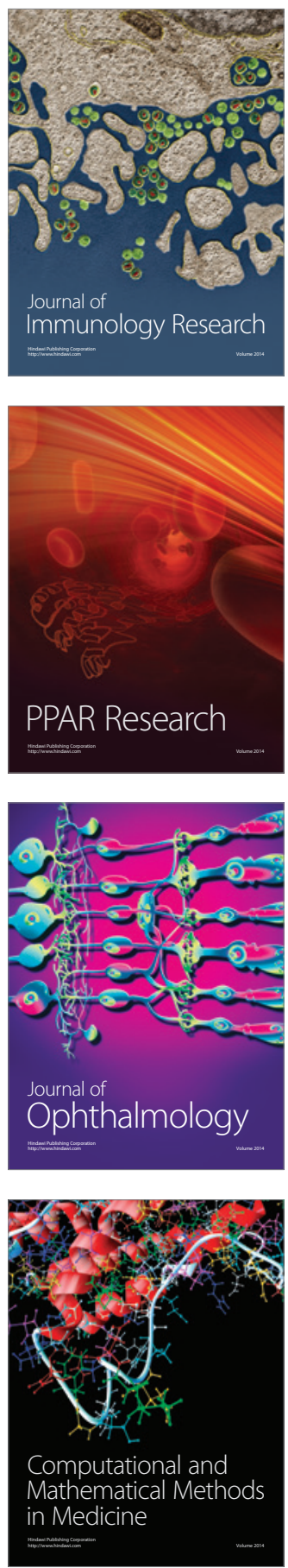

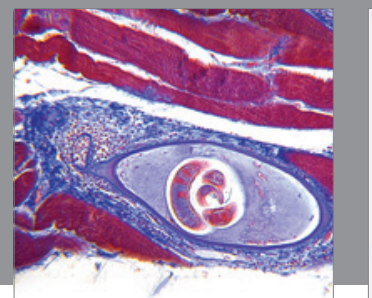

Gastroenterology

Research and Practice
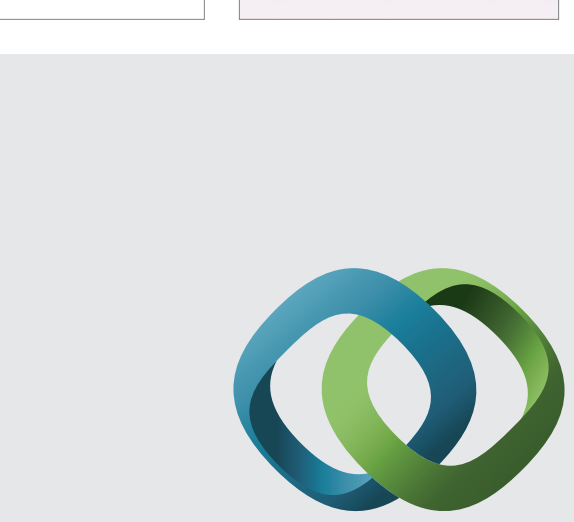

\section{Hindawi}

Submit your manuscripts at

http://www.hindawi.com
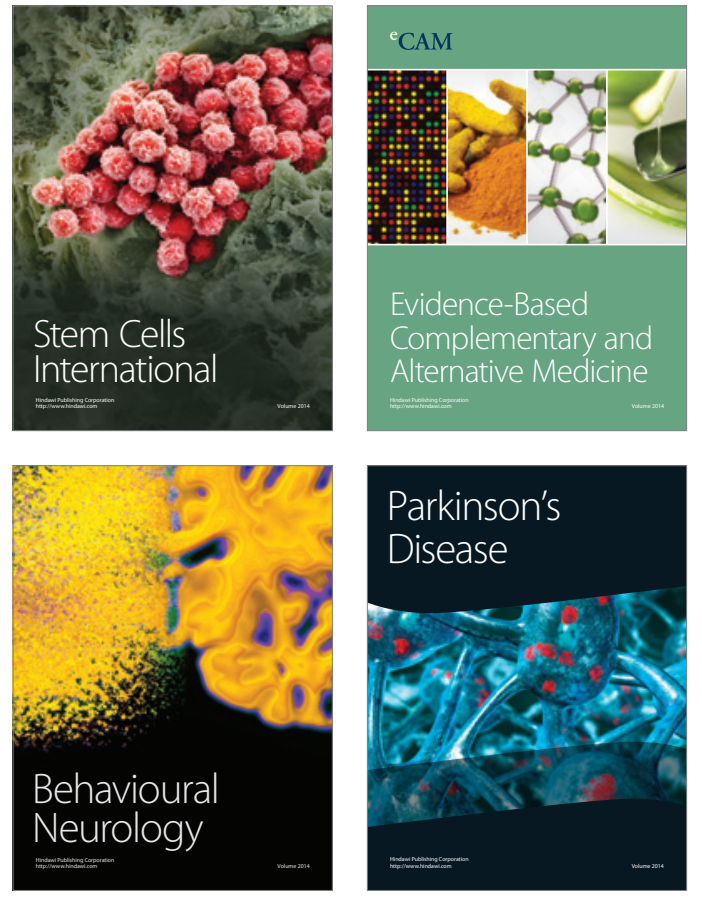
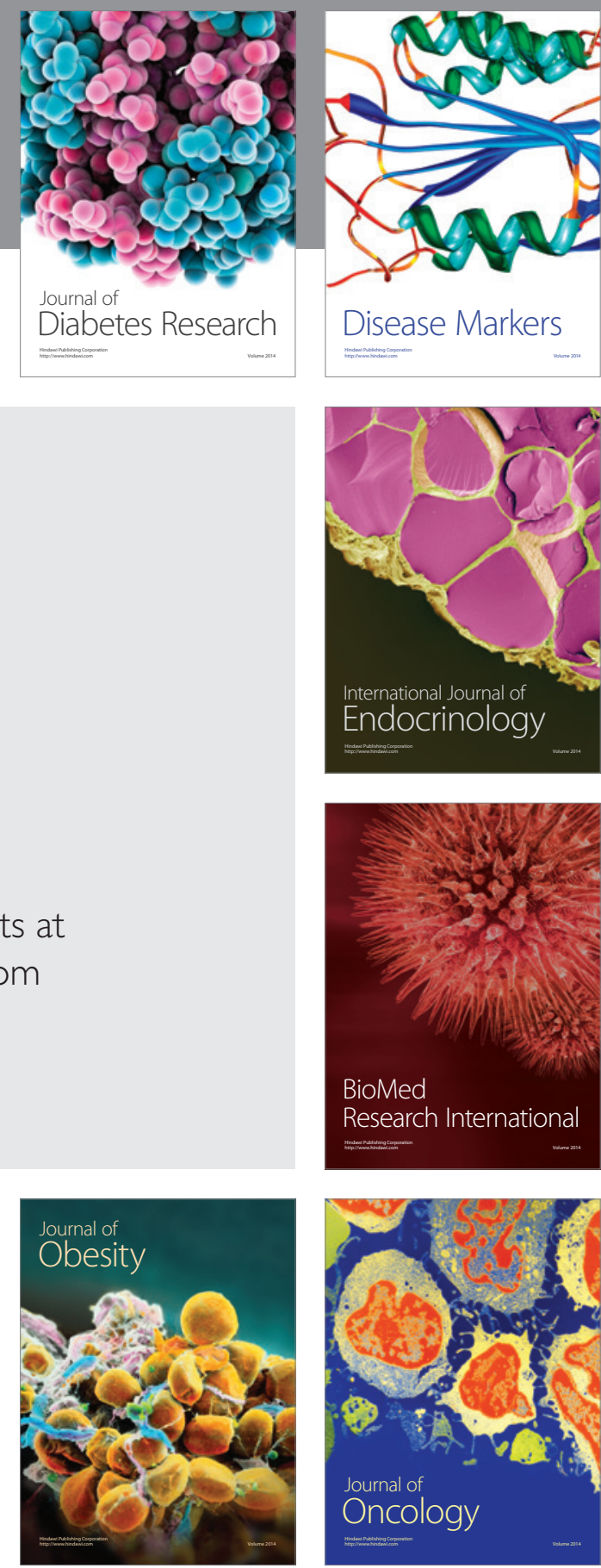

Disease Markers
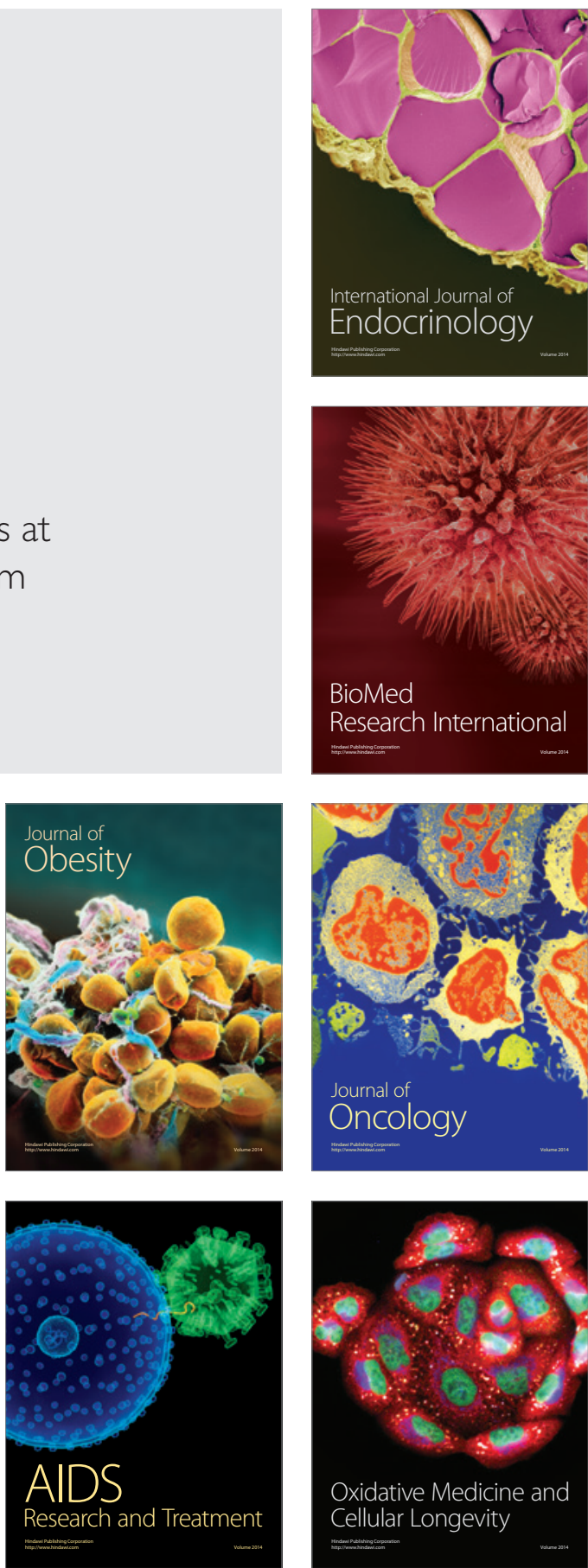